Elsevier required licence: (c) <2019>. This manuscript version is made available under the CC-BY-NC-ND 4.0 license http://creativecommons.org/licenses/by-nc-nd/4.0/

The definitive publisher version is available online at https://doi.org/10.1016/j.compstruct.2018.12.031 


\title{
Dynamic multiscale topology optimization for multi- regional micro-structured cellular composites
}

\author{
Jie Gao ${ }^{a}$,, Zhen Luo ${ }^{b}$, Hao Li ${ }^{\text {a }}$, Peigen $\mathrm{Li}^{\mathrm{a}}$, *Liang Gao

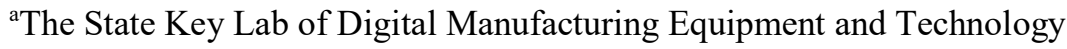 \\ Huazhong University of Science and Technology, 1037 Luoyu Road, Wuhan, Hubei 430074, China \\ ${ }^{\mathrm{b}}$ The School of Mechanical and Mechatronic Engineering \\ University of Technology Sydney, 15 Broadway, Ultimo, NSW 2007, Australia \\ * Corresponding author: Tel.: +86-27-87557742; E-mail: gaoliang@mail.hust.edu.cn (Prof L. Gao)
}

\begin{abstract}
In this paper, a new dynamic multiscale topology optimization method for cellular composites with multiregional material microstructures is proposed to improve the structural performance. Firstly, a free-material distribution optimization method (FMDO) is developed to generate the overall configuration for the discrete element densities distributed within a multi-regional pattern. The macrostructure is divided into several sub regions, and each of them consists of a number of elements but with the same densities. Secondly, a dynamic topology optimization formulation is developed to perform the concurrent design of the macrostructure and material microstructures, subject to the multi-regional distributed element densities. A parametric level set method is employed to optimize the topologies of the macrostructure and material microstructures, with the effective macroscopic properties evaluated by the homogenization. In the numerical implementation, the quasi-static Ritz vector (QSRV) method is incorporated into the finite element analysis so as to reduce the computational cost in numerical analysis, and some kinematical connectors are introduced to make sure the connectivity between adjacent material microstructures. Finally, 2D and 3D numerical examples are tested to demonstrate the effectiveness of the proposed dynamic multiscale topology optimization method for the material-structural composites.
\end{abstract}

Keywords: Multiscale topology optimization; Cellular composites; Dynamic compliance; Level set method; Microstructures. 


\section{Introduction}

Cellular composites are a kind of artificially architected materials with the solids and voids, with extensive applications in engineering owing to their multifunctional properties [1,2], such as the honeycomb cellular composites having been considerably applied into the industry [3]. Moreover, how to improve the structural dynamic characteristics, like the vibration control and the reduction of frequency responses, has become an important topic in the field [4,5]. There are a large number of studies focused on the structural optimization of cellular composites under static loads $[1,2]$, but only a limited number of works have been reported for multiscale topology optimization of cellular composites considering the vibration.

In structural optimization, topology optimization has becoming very popular over the past two decades, due to its capability in finding an overall framework for the conceptual design without prior knowledge. Many different methods have been developed for topology optimization in recent years, like the homogenization method [6], the solid isotropic material with penalization (SIMP) $[7,8]$ method, the evolutionary structural optimization (ESO) [9], the level set method (LSM) [10-12], and the phase-field method, e.g. [13,14]. One of them, LSM offers unique characteristics by evolving geometrical boundaries to implement shape and topological changes rather than updating element densities $[11,12]$.

It is important to note that several complex numerical issues have restrained the further applications of the LSM to more advanced design problems [11,12]. Hence, many variant LSM methods have been developed in order to eliminate the numerical difficulties, e.g. [15-20]. Particularly, the parametrization LSM (PLSM) $[16,17]$ has been demonstrated as one of efficient methods for structural optimization. It can not only inherit the favorable features of the most conventional LSMs, but also eliminate the complex numerical issues due to the implicit surface interpolation by the compactly supported basis functions (CSRBFs) [21]. Hence, the evolution of the structural boundaries is then achieved by iteratively updating the expansion coefficients of the interpolant. That is, the numerical implementation after the interpolation of the higher-dimension level set function is similar to the SIMP but interpolation points rather than elements based. Moreover, the OC (optimality criteria) method [22] and the method of moving asymptotes (MMA) [23] can be directly used to solve the optimization problems. The PLSM and its variants have shown the generality and applicability for different design problems [24,25].

Topology optimization has also been applied to the architected materials design for the generation of novel materials and even nanostructures, to achieve the extreme or prescribed material properties for the cellular composites [26,27]. Since the inverse homogenization method is proposed in [28], many different topology 
optimization methods combined with the homogenization have been developed for a wide range of designs of cellular composites [29-35]. However, it should be noted that the above works for topology optimization focused on the monoscale designs.

In pursuing the higher structural performance, the concurrent design idea has been introduced into topology optimization with the considerations of both the macro topology and the effective macroscopic properties $[36,37]$. The earlier works perform the optimization of material microstructure in a pre-defined distribution within the macrostructure [38]. After that, the concurrent topology optimization for the macrostructure and material microstructure has been discussed by many topology optimization methods [39,40], where only a unique material microstructure is configured in the macrostructure. This design can ensure the connectivity and remarkably reduces the computational cost, while the ultimate intention for the structural performance is strongly compromised. Later, multiscale topology optimization for cellular composites with the multiple microstructures is also studied [41-50], while the computational cost is prohibitive due to the optimization of a large number of microstructures.

Since the first work for the dynamic [4], many dynamic problems have been discussed, like the maximizing fundamental eigenfrequency [5,51] and frequency response optimization [52-54]. The natural frequency problem intends to drive the fundamental eigenfrequency away from the excitation frequency to avoid the resonance, while the main intention of the frequency response problems is to reduce the response over a part of the structure or the whole domain. Ma et al [5] applied the homogenization method to improve the performance under a definition of the structural dynamic compliance. Then, the LSM has also been applied to minimize the local or global frequency response [53,54]. After that, a generalized incremental frequency method to minimize the dynamic compliance was proposed by Olhoff et al [52], subject to the prescribed low or high value of the excitation frequencies. Many model reduction schemes have also been developed to save the computational demands in the dynamic topology optimization $[55,56]$. It can be found that all the aforementioned works only consider the optimization at the macroscale.

In order to improve the dynamic performance, the dynamic multiscale topology optimization for the design of cellular composites has been discussed [57], where the topologies of both the macrostructure and material microstructure are concurrently optimized to the maximum fundamental frequency. Then, the BESO is used to develop a concurrent topology optimization model to maximize the fundamental frequency [58]. Xu et al [59] performed the concurrent design of the composite macrostructures and multi-phase microstructure for minimizing the structural dynamic compliance. However, it should be noted that the previous works for 
the dynamic multiscale topology optimization only considered a unique microstructure configured in the macro domain. In order to further enhance the dynamic performance, it is of great importance to investigate the dynamic topology optimization for cellular composites with multiple material microstructures.

Hence, this research is motivated to develop a new dynamic topology optimization method for multiscale design of the cellular composites with multiple microstructures. Firstly, a FMDO formulation [60] will be employed to generate an overall distribution of multiple element densities in the macro design domain, so that the macro design domain is divided into several sub regions with the discrete densities. Each sub region is homogenously configured by a kind of the identical densities, which corresponds to the specific effective macroscopic property of a representative microstructure. Secondly, the topology of the macrostructure and the topologies of multiple microstructures are concurrently optimized to minimize the dynamic compliance, subject to the multi-regional distribution of the discrete element densities. Finally, two numerical examples will be performed to demonstrate the effectiveness of the proposed method.

\section{Finite element analysis for the dynamic}

The momentum equation of structures with viscous damping can be formulated by Newton's law [5] when considering a dynamic external load, given as:

$$
\mathbf{M} \ddot{\mathbf{U}}_{t}+\dot{\mathbf{C U}}_{t}+\mathbf{K} \mathbf{U}_{t}=\mathbf{F}_{t}
$$

where $\mathbf{M}, \mathbf{C}$ and $\mathbf{K}$ are the mass, damping and stiffness matrices for structures, respectively. The global displacement, velocity and acceleration field in the design domain varied with time are denoted by $\mathbf{U}_{t}, \dot{\mathbf{U}}_{t}$

and $\ddot{\mathbf{U}}_{t}$, respectively. $\mathbf{F}_{t}$ is the loaded external load vector. A Rayleigh damping model [5] is applied to evaluate the damping matrix by a linear combination of the mass and stiffness matrices, as:

$$
\mathbf{C}=\mathcal{A M}+\mathcal{B} \mathbf{K}
$$

where $\mathcal{A}$ and $\mathcal{B}$ are the related damping coefficients, respectively. To obtain the structural responses by the defined harmonic load with an excitation frequency $\omega$, the dynamic force and displacement field can be written in an exponential form, as:

$$
\left\{\begin{array}{l}
\mathbf{U}_{t}=\mathrm{U} e^{i \omega t} \\
\mathbf{F}_{t}=\mathrm{F} e^{i \omega t}
\end{array}\right.
$$

where $U$ and $F$ are the amplitudes of the external load and the displacement, respectively. Eq. (1) can be written as a new form of the amplitudes of the displacement response and the applied force, when only considering the frequency domain, given as: 


$$
\left(-\omega^{2} \mathbf{M}+i \omega \mathbf{C}+\mathbf{K}\right) \mathrm{U}=\mathrm{F}
$$

For simplicity, Eq. (4) can be written in a compact form by the dynamic stiffness matrix, which is defined by the assembly of the mass, damping and stiffness matrices, denoted by $\mathbf{K}_{d}$ :

$$
\left\{\begin{array}{l}
\mathbf{K}_{d}=-\omega^{2} \mathbf{M}+i \omega \mathbf{C}+\mathbf{K} \\
\mathrm{F}=\mathbf{K}_{d} \mathrm{U}
\end{array}\right.
$$

\section{Parametric level set method for cellular composites}

A simple illustration of a 3D cellular composite at two scales is shown in Fig. 1, where the macrostructure is depicted in the global coordinate system $\mathbf{x}$, and the local coordinate system $\mathbf{y}$ is applied to describe the microstructures. It can be easily seen that the macrostructure contains two different microstructures, which are respectively distributed in their corresponding locations in the macro domain.

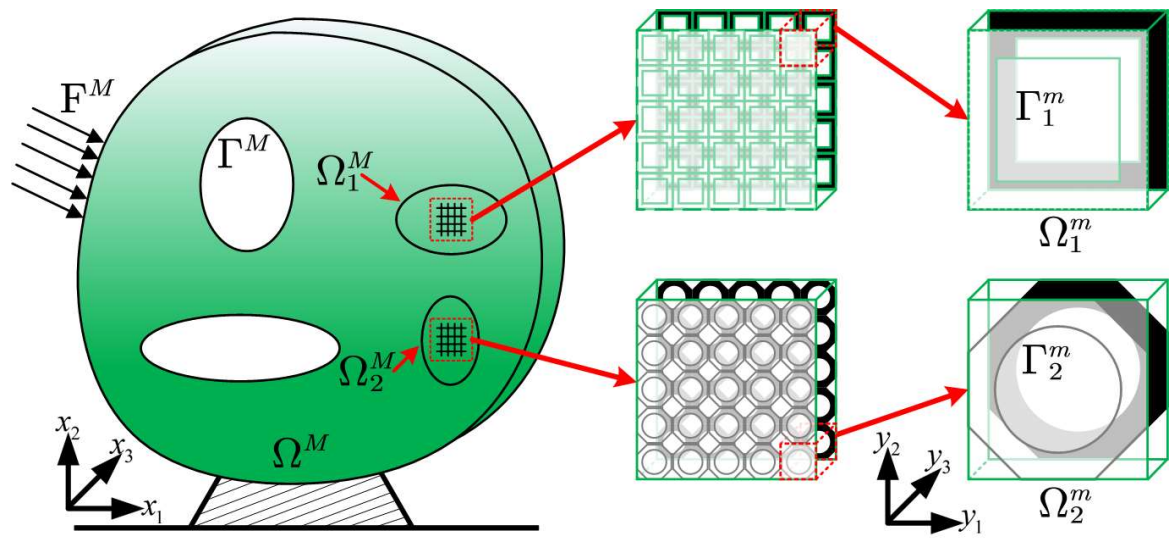

(a) The macrostructure

(b) Material microstructures

Fig. 1. 3D Cellular composite at two scales

It is noted that the superscript $M$ indicates the macroscale quantities, and $m$ is related to the quantities at the micro scale. In the LSM, the implicit dynamic surface is utilized to represent the structural topology, in which the boundary of the structure is implicitly described by the zero-level set of a higher-dimensional level set function (LSF) [10], as given in Fig. 2. As far as cellular composites with multiple microstructures, several LSFs are required to represent the macrostructure and material microstructures, respectively.

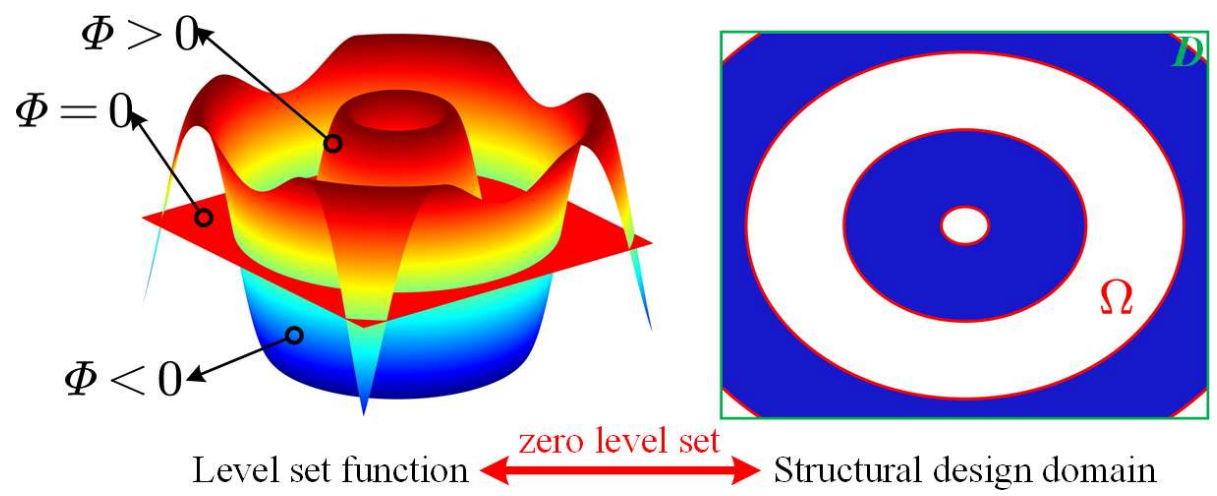

Fig. 2. 3D LSF and 2D structural design domain 
A higher-dimensional level set function is firstly defined to represent the macro topology, given as:

$$
\begin{cases}\Phi^{M}(\mathbf{x})>0, & \forall \mathbf{x} \in \Omega^{M} / \Gamma^{M} \quad \text { (macro solid) } \\ \Phi^{M}(\mathbf{x})=0, & \forall \mathbf{x} \in \Omega^{M} \cap \Gamma^{M} \quad \text { (macro boundary) } \\ \Phi^{M}(\mathbf{x})<0, & \forall \mathbf{x} \in D^{M} / \Omega^{M} \quad \text { (macro void) }\end{cases}
$$

where $\Omega^{M}, \Gamma^{M}$ and $D^{M}$ denote the design domain, structural boundary and reference domain of the macrostructure, respectively. Here, Assuming that there exist $\Theta$ distinct material microstructures in the porous composite $(\Theta=2$ in Fig. 1), we should introduce $\Theta$ LSFs to represent the microstructures, respectively, expressed as:

$$
\left\{\begin{array}{llc}
\Phi_{\vartheta}^{m}(\mathbf{y})>0, & \forall \mathbf{y} \in \Omega_{\vartheta}^{m} / \Gamma_{\vartheta}^{m} & \text { (micro solid) } \\
\Phi_{\vartheta}^{m}(\mathbf{y})=0, & \forall \mathbf{y} \in \Omega_{\vartheta}^{m} \cap \Gamma_{\vartheta}^{m} \quad \text { (micro boundary) } \quad\{\vartheta=1,2, \cdots, \Theta\} \\
\Phi_{\vartheta}^{m}(\mathbf{y})<0, & \forall \mathbf{y} \in D_{\vartheta}^{m} / \Omega_{\vartheta}^{m} \quad \text { (micro void) }
\end{array}\right.
$$

where $\Omega_{\vartheta}^{m}, \Gamma_{\vartheta}^{m}$ and $D_{\vartheta}^{m}$ indicate the design domain, structural boundary and reference domain of the $\vartheta_{t h}$ representative material microstructure, respectively. Introducing a pseudo-time into Eqs. (6) and (7), and differentiating them on both sides with respect to the time variable $t$, the dynamic boundaries at two scales can be expressed through the H-J PDEs [11,12], expressed as:

$$
\left\{\begin{array}{l}
\frac{\partial \Phi^{M}}{\partial t}-v_{\mathbf{n}}^{M}\left|\nabla \Phi^{M}\right|=0 \\
\frac{\partial \Phi_{\vartheta}^{m}}{\partial t}-v_{\vartheta, \mathbf{n}}^{m}\left|\nabla \Phi_{\vartheta}^{m}\right|=0
\end{array}\right.
$$

where $v_{\mathbf{n}}^{M}$ and $v_{\vartheta, \mathbf{n}}^{m}$ denote the corresponding normal velocity fields at two scales, respectively. Hence, the advancing of the structural boundaries by the normal velocity fields corresponds to the feasible solutions of the H-J PDEs. However, solving the H-J PDEs requires the complicated numerical schemes $[11,12]$.

In the PLSM $[16,17]$, the LSF is interpolated by a set of centrally positioning radially symmetric CSRBFs at a number of knots over the design domain. Hence, the LSFs at two scales are defined as:

$$
\left\{\begin{array}{l}
\Phi^{M}(\mathbf{x}, t)=\boldsymbol{\varphi}^{T}(\mathbf{x}) \boldsymbol{\alpha}^{M}(t)=\sum_{i=1}^{N_{M}} \varphi_{i}(\mathbf{x}) \alpha_{i}^{M}(t) \\
\Phi_{\vartheta}^{m}(\mathbf{y}, t)=\boldsymbol{\varphi}^{T}(\mathbf{y}) \boldsymbol{\alpha}_{\vartheta}^{m}(t)=\sum_{j=1}^{N_{m}} \varphi_{j}(\mathbf{y}) \alpha_{\vartheta, j}^{m}(t)
\end{array}\right.
$$

where $N_{M}$ and $N_{m}$ are the total number of the CSRBF knots in the macrostructure and microstructures respectively, and the CSRBFs with $\mathrm{C}^{2}$ continuity [21] is employed here:

$$
\varphi(r)=(1-r)_{+}^{4}(4 r+1)
$$


where $r$ is used to control the size of the influence domain of the basis function at the CSRBFs knot. The H-J PDEs can be expanded as the new forms by substituting Eq. (9) into Eq. (8), given as:

$$
\left\{\begin{array}{l}
\boldsymbol{\varphi}^{T}(\mathbf{x}) \frac{d \boldsymbol{\alpha}^{M}(t)}{d t}-v_{\mathbf{n}}^{M}\left|\nabla \boldsymbol{\varphi}^{T}(\mathbf{x}) \boldsymbol{\alpha}^{M}(t)\right|=0 \\
\boldsymbol{\varphi}^{T}(\mathbf{y}) \frac{d \boldsymbol{\alpha}_{\vartheta}^{m}(t)}{d t}-v_{\vartheta, \mathbf{n}}^{m}\left|\nabla \boldsymbol{\varphi}^{T}(\mathbf{y}) \boldsymbol{\alpha}_{\vartheta}^{m}(t)\right|=0
\end{array}\right.
$$

and the normal velocities $v_{\mathbf{n}}^{M}$ and $v_{\vartheta, \mathbf{n}}^{m}$ at two scales can then be given as:

$$
\left\{\begin{array}{l}
v_{\mathbf{n}}^{M}=\frac{\boldsymbol{\varphi}^{T}(\mathbf{x})}{\left|\nabla \boldsymbol{\varphi}^{T}(\mathbf{x}) \boldsymbol{\alpha}^{M}(t)\right|} \frac{d \boldsymbol{\alpha}^{M}(t)}{d t} \\
v_{\vartheta, \mathbf{n}}^{m}=\frac{\boldsymbol{\varphi}^{T}(\mathbf{y})}{\left|\nabla \boldsymbol{\varphi}^{T}(\mathbf{y}) \boldsymbol{\alpha}_{\vartheta}^{m}(t)\right|} \frac{d \boldsymbol{\alpha}_{\vartheta}^{m}(t)}{d t}
\end{array}\right.
$$

As we can see, the normal velocities at two scales have been naturally applied to the corresponding domains due to the CSRBFs positioned in the whole design domain. The topology optimization problems driven by the H-J PDEs have been re-shaped into the ordinary differential equations (ODEs), even algebraic equations (AEs), with the interpolation coefficients unknown [16,17].

\section{Dynamic multiscale topology optimization for cellular composites}

The procedure of the current dynamic multiscale topology optimization formulation for cellular composites is illustrated in Fig. 3, which involves the stage of the FMDO and then the stage of the concurrent topology optimization. The initial structural design domain is displayed in Fig. 3 (a).

As shown in Fig. 3 (b), a continuous distribution (given in the first figure) of the element densities is firstly obtained by the FMDO formulation. It can be seen that a large number of the intermediate element densities are existed in the macro domain, which results in a huge amount of material microstructures to be designed. In this case, the computational cost is prohibitive for the latter concurrent topology optimization. Hence, a regularization mechanism needs to be defined to reduce the number of the intermediate element densities. The regularized distribution of the element densities is given in Fig. $3(b)$. As we can see, the regularized element densities are distributed in a discrete and multi-regional manner, in which the macro design domain is divided into several sub regions and each sub region is configured with a unique density but with a large number of elements.

Then, the topology of the macrostructure and the topologies of multiple microstructures are optimized in a concurrent topology optimization procedure to minimize dynamic compliance, subject to the multi-regional 
distribution of the discrete element densities. At the macro, the PLSM is applied to optimize the topology of the macrostructure under the global volume constraint, displayed in Fig. 3 (c). At the micro, the topology of each material microstructure is also optimized by the PLSM with the numerical homogenization method, subject to the local volume constraint defined by the discrete element density. The topologies for multiple microstructures are indicated in Fig. 3 (c). The final multiscale design of the cellular composite is displayed in Fig. $3(d)$. It can be easily found that the optimized topology of the macrostructure is divided into three sub regions plotted with different colors (white, red and black), and each sub region is uniformly configured by the representative material microstructure with the same color. Hence, the dynamic multiscale topology design of the cellular composite with multi-regional microstructures can be obtained.

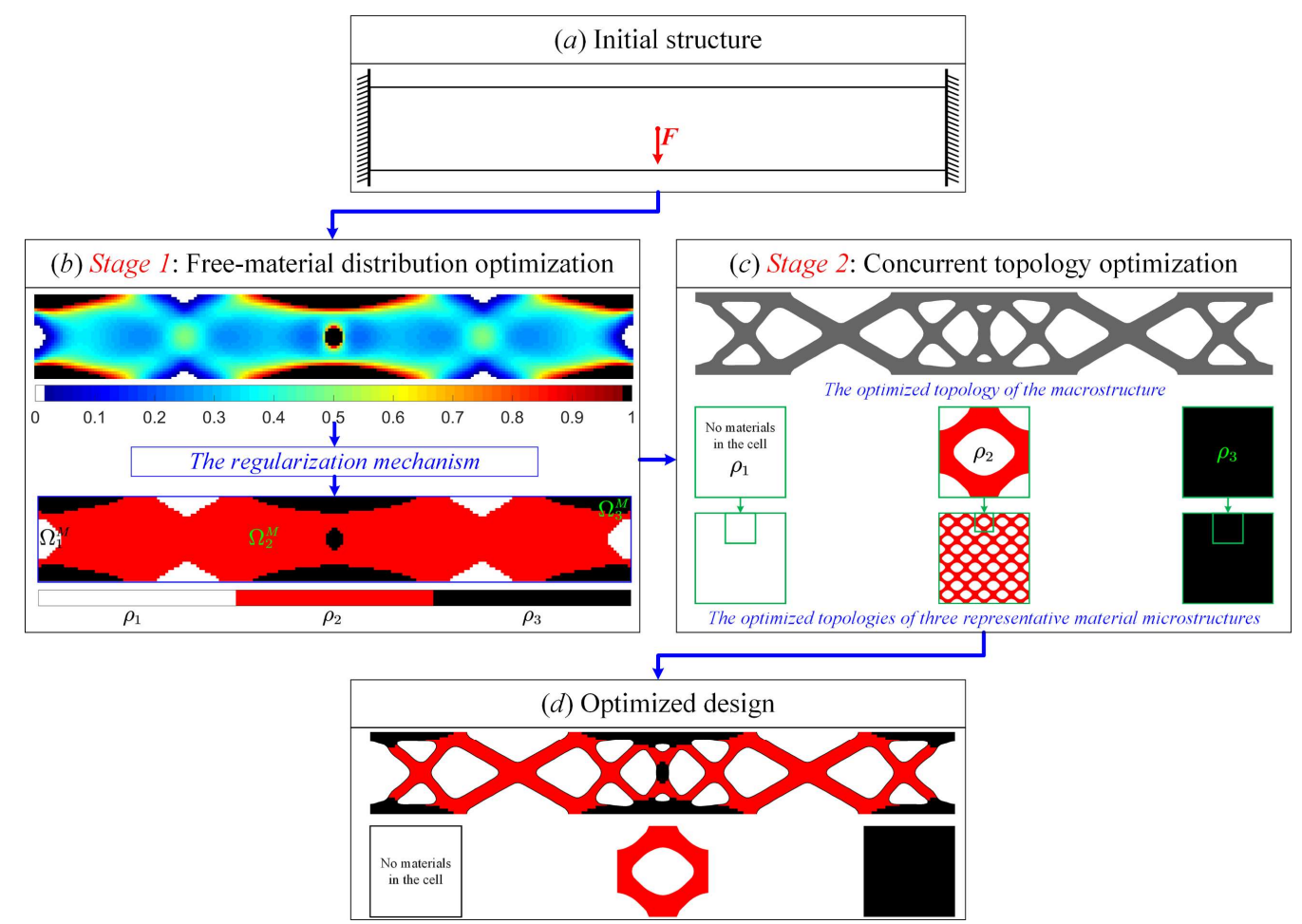

Fig. 3. Dynamic multiscale topology optimization for cellular composites

\subsection{Free-material distribution optimization (FMDO)}

The FMDO [60] is employed here to optimize the distribution of the material element densities to minimize the dynamic compliance, and the mathematical form is expressed as:

$$
\left\{\begin{array}{l}
\text { Find: } \boldsymbol{\rho}=\left\{\rho_{1}, \rho_{2}, \cdots, \rho_{e}, \cdots, \rho_{N_{e}}\right\} \\
\text { Min: } J_{d}=|J|=\left|\mathbf{F}^{T} \mathbf{U}\right| \\
\text { S.t: }\left\{\begin{array}{l}
\mathbf{K}_{d} \mathbf{U}=\mathbf{F} \\
G_{d}=\sum_{e=1}^{N_{e}} \rho_{e} v_{0}-V_{d} \leq 0 \\
0<\rho_{\min } \leq \rho_{e} \leq 1\left(e=1,2, \cdots, N_{e}\right)
\end{array}\right.
\end{array}\right.
$$


where $J$ is the dynamic compliance. As described in [5], the dynamic compliance is a complex number. The real and image parts of $J$ are symbolled by $J_{R}$ and $J_{I}$, respectively. The module of the dynamic compliance $J_{d}$ acts as the objective function for numerical stability, defined as:

$$
J_{d}=\sqrt{\left(J_{R}\right)^{2}+\left(J_{I}\right)^{2}}
$$

where $\mathbf{F}$ is the external dynamic load vector with a certain excitation frequency $\omega$, and $\mathbf{U}$ is the global displacement field. $\mathbf{K}_{d}$ is the global dynamic stiffness matrix defined by Eq. (5). $G_{d}$ denotes the volume constraint subject to the maximum volume fraction $V_{d} . v_{0}$ is the volume fraction of solid elements. $\rho_{e}$ is the $e_{t h}$ element density ranging from 0 to $1 . N_{e}$ is the total number of the finite elements to discretize the macro design domain. The formulation in Eq. (13) is analogous to the classic SIMP method [7,8], while the penalty mechanism is not employed to ensure a black-white design. Hence, the phenomenon of the localized modes does not appear in the optimization [52-54]. The formulation in Eq. (14) can generate a continuous distribution of the element densities, with a large number of the intermediate densities. The regularization mechanism is developed to remarkably reduce the number of intermediate element densities. It is assumed that the element densities in the continuous distribution are classified into $\Theta$ groups, given as:

$$
\bar{\rho}_{\vartheta}=\frac{1}{N_{\vartheta}} \sum_{i=1}^{N_{\xi}} \rho_{\vartheta}^{i} \quad\left(\rho_{\vartheta}^{\min } \leq \rho_{\vartheta}^{i}<\rho_{\vartheta}^{\max }, \vartheta=1,2, \cdots, \Theta\right)
$$

where $\rho_{\vartheta}^{i}$ is the $i_{t h}$ element density belonging to the $\vartheta_{t h}$ group. $\rho_{\vartheta}^{\min }$ and $\rho_{\vartheta}^{\max }$ are the defined lower and upper thresholds of the element densities in the $\vartheta_{t h}$ group, respectively. $N_{\vartheta}$ is the total number of elements in the $\vartheta_{t h}$ group, and $\bar{\rho}_{\vartheta}$ is the regularized density of the $\vartheta_{t h}$ group which is defined by the average of all element densities in this group. The macro domain will also be divided into $\Theta$ different sub regions, namely $\Omega_{1}^{M}, \Omega_{2}^{M}, \cdots, \Omega_{\vartheta}^{M}, \cdots, \Omega_{\Theta}^{M}$.

\subsection{Concurrent topology optimization to minimize the dynamic compliance}

The latter stage in the multiscale design for porous composites aims to optimize the topology of the macrostructure and the topologies of multiple material microstructures, subject to the multi-regional distribution of the discrete element densities achieved in the former stage, namely the FMDO. In this work, the PLSM $[16,17]$ is applied to optimize the topologies at two scales, with the numerical homogenization method [61] to evaluate the effective macroscopic properties. The detailed mathematical model for the concurrent design of the macrostructure and multiple material microstructures is expressed as: 


$$
\left\{\begin{array}{l}
\text { Find: } \alpha_{i}^{M}, \alpha_{\vartheta, j}^{m} \quad\left(i=1,2, \cdots, N_{M} ; \vartheta=1,2, \cdots, \Theta ; j=1,2, \cdots, N_{m} ;\right) \\
\operatorname{Min}_{\mathbf{u}, \Phi}: J_{d}(\mathbf{u}, \Phi)=|J(\mathbf{u}, \Phi)|=\left|\sum_{\vartheta=1}^{\Theta} \int_{D^{M}} \mathbf{F}^{T} \mathbf{u}_{\vartheta}^{M} H\left(\Phi_{\vartheta}^{M}\right) d \Omega_{\vartheta}^{M}\right| \\
\text { S.t: }\left\{\begin{array}{l}
a\left(\Phi^{M}, \mathbf{u}^{M}, \mathbf{v}^{M}, \mathbf{E}^{H}\right)=l\left(\Phi^{M}, \mathbf{v}^{M}\right), \quad \forall \mathbf{v}^{M} \in \overline{\mathrm{U}}\left(\Omega^{M}\right) \\
a\left(\Phi_{\vartheta}^{m}, \mathbf{u}_{\vartheta}^{m}, \mathbf{v}_{\vartheta}^{m}\right)=l\left(\Phi_{\vartheta}^{m}, \mathbf{v}_{\vartheta}^{m}\right), \quad \forall \mathbf{v}_{\vartheta}^{m} \in \overline{\mathrm{U}}\left(\Omega_{\vartheta}^{m}\right) \\
G_{M}=\sum_{\vartheta=1}^{\Theta}\left\{\int_{D^{M}} H\left(\Phi_{\vartheta}^{M}\right) d \Omega_{\vartheta}^{M} \int_{D_{\vartheta}^{m}} H\left(\Phi_{\vartheta}^{m}\right) d \Omega_{\vartheta}^{m}\right\} \\
G_{\vartheta}^{m}=\int_{D_{\vartheta}^{m}} H\left(\Phi_{\vartheta}^{m}\right) d \Omega_{\vartheta}^{m}-\bar{\rho}_{\vartheta} \leq 0 \\
\alpha_{\min }^{M} \leq \alpha_{i}^{M} \leq \alpha_{\text {max }}^{M} ; \alpha_{\vartheta, \min }^{m} \leq \alpha_{\vartheta, j}^{m} \leq \alpha_{\vartheta, \max }^{m}
\end{array}\right.
\end{array}\right.
$$

where $\alpha_{i}^{M}$ is the $i_{t h}$ macro design variable of the macrostructure bounded by $\alpha_{\min }^{M}$ and $\alpha_{\max }^{M} \cdot \alpha_{\vartheta \text {,min }}^{m}$ and $\alpha_{\vartheta, \text { max }}^{m}$ are the lower and upper bounds of the design variable $\alpha_{\vartheta, j}^{m}$ for $\vartheta_{t h}$ material microstructure, respectively. $J$ is the structural dynamic compliance, and the objective function is defined by the module of the dynamic compliance, denoted by $J_{d} . G_{M}$ is the global volume constraint calculated by considering the macrostructure and the representative microstructures. $V_{M}$ is the total material consumption. $G_{\vartheta}^{m}$ is the volume constraint of the $\vartheta_{t h}$ microstructure, which is equal to the regularized density $\bar{\rho}_{\vartheta}$. $H$ is the Heaviside function [10]. $\mathbf{u}^{M}$ is the macro displacement field, and $\mathbf{v}^{M}$ is the macro virtual displacement field belonging to the kinematically admissible space $\overline{\mathrm{U}}\left(\Omega^{M}\right)$. The macro equilibrium equation for the dynamic compliance is developed by the bilinear energy term $a$ and the load linear term $l$, given as:

$$
\left\{\begin{array}{l}
a=\sum_{\vartheta=1}^{\Theta} k\left(\Phi_{\vartheta}^{M}, \mathbf{u}_{\vartheta}^{M}, \mathbf{v}_{\vartheta}^{M}, \mathbf{E}_{\vartheta}^{H}\right)+i \omega c\left(\Phi_{\vartheta}^{M}, \mathbf{u}_{\vartheta}^{M}, \mathbf{v}_{\vartheta}^{M}, \mathbf{E}_{\vartheta}^{H}\right)-\omega^{2} m\left(\Phi_{\vartheta}^{M}, \mathbf{u}_{\vartheta}^{M}, \mathbf{v}_{\vartheta}^{M}, \mathbf{E}_{\vartheta}^{H}\right) \\
l=\int_{D^{M}} p \mathbf{v}^{M} H\left(\Phi^{M}\right) d \Omega^{M}+\int_{D^{M}} f \mathbf{v}^{M} \delta\left(\Phi^{M}\right)\left|\nabla \Phi^{M}\right| d \Omega^{M}
\end{array}\right.
$$

where $p$ is the macro body force and $f$ is the macro boundary traction. $\delta$ is the partial derivative of the Heaviside function $H . k, c$ and $m$ indicate the stiffness, the damping, the mass and the load functions, respectively, expressed by:

$$
\left\{\begin{array}{l}
k\left(\Phi_{\vartheta}^{M}, \mathbf{u}_{\vartheta}^{M}, \mathbf{v}_{\vartheta}^{M}, \mathbf{E}_{\vartheta}^{H}\right)=\int_{D^{M}} \boldsymbol{\varepsilon}\left(\mathbf{u}_{\vartheta}^{M}\right) \mathbf{E}_{\vartheta}^{H}\left(\mathbf{u}_{\vartheta}^{M}, \Phi_{\vartheta}^{M}\right) \boldsymbol{\varepsilon}\left(\mathbf{v}_{\vartheta}^{M}\right) H\left(\Phi_{\vartheta}^{M}\right) d \Omega^{M} \\
m\left(\Phi_{\vartheta}^{M}, \mathbf{u}_{\vartheta}^{M}, \mathbf{v}_{\vartheta}^{M}, \mathbf{E}_{\vartheta}^{H}\right)=\int_{D^{M}} \psi_{\vartheta}^{H} \mathbf{u}_{\vartheta}^{M} \mathbf{v}_{\vartheta}^{M} H\left(\Phi_{\vartheta}^{M}\right) d \Omega^{M} \\
c\left(\Phi_{\vartheta}^{M}, \mathbf{u}_{\vartheta}^{M}, \mathbf{v}_{\vartheta}^{M}, \mathbf{E}_{\vartheta}^{H}\right)=\mathcal{A} m\left(\Phi_{\vartheta}^{M}, \mathbf{u}_{\vartheta}^{M}, \mathbf{v}_{\vartheta}^{M}, \mathbf{E}_{\vartheta}^{H}\right)+\mathcal{B} k\left(\Phi_{\vartheta}^{M}, \mathbf{u}_{\vartheta}^{M}, \mathbf{v}_{\vartheta}^{M}, \mathbf{E}_{\vartheta}^{H}\right)
\end{array}\right.
$$

where $\psi_{\vartheta}^{H}$ is the homogenized mass density of the $\vartheta_{t h}$ representative microstructure, and the simple but efficient "ersatz material" model [12] is applied to evaluate it, given by: 


$$
\psi_{\vartheta}^{H}=\frac{1}{\left|\Omega_{\vartheta}^{m}\right|} \int_{D_{\vartheta}^{m}} \psi_{0} H\left(\Phi_{\vartheta}^{m}\right) d \Omega_{\vartheta}^{m}
$$

where $\psi_{0}$ is the mass density of the material. $\mathbf{E}_{\vartheta}^{H}$ denotes the homogenized elasticity tensor of the $\vartheta_{t h}$ representative microstructure evaluated by the homogenization [61], as:

$$
E_{\vartheta(i j k l)}^{H}=\frac{1}{\left|\Omega_{\vartheta}^{m}\right|} \int_{D_{\vartheta}^{m}}\left(\varepsilon_{p q}^{0(i j)}-\varepsilon_{p q}^{*}\left(u_{\vartheta}^{m(i j)}\right)\right) E_{p q r s}\left(\varepsilon_{r s}^{0(k l)}-\varepsilon_{r s}^{*}\left(u_{\vartheta}^{m(k l)}\right)\right) H\left(\Phi_{\vartheta}^{m}\right) d \Omega_{\vartheta}^{m}
$$

where $E_{p q r s}$ is the constituent elasticity property of the material. $\varepsilon_{p q}^{0(i j)}$ is the initial unit test strain. $\varepsilon_{p q}^{*}$ is the locally varying strain field induced by $\varepsilon_{p q}^{0(i j)}$. The unknown displacement field $u_{\vartheta}^{m(i j)}$ is calculated by solving the micro elastic equilibrium equation, given as:

$$
\left\{\begin{array}{l}
a\left(\Phi_{\vartheta}^{m}, \mathbf{u}_{\vartheta}^{m}, \mathbf{v}_{\vartheta}^{m}\right)=\int_{D_{\vartheta}^{m}} \varepsilon_{p q}^{*}\left(u_{\vartheta}^{m(i j)}\right) E_{p q r s} \varepsilon_{r s}^{*}\left(v_{\vartheta}^{m(k l)}\right) H\left(\Phi_{\vartheta}^{m}\right) d \Omega_{\vartheta}^{m} \\
l\left(\Phi_{\vartheta}^{m}, \mathbf{v}_{\vartheta}^{m}\right)=\int_{D_{\vartheta}^{m}} \varepsilon_{p q}^{0(i j)} E_{p q r s} \varepsilon_{r s}^{*}\left(v_{\vartheta}^{m(k l)}\right) H\left(\Phi_{\vartheta}^{m}\right) d \Omega_{\vartheta}^{m}
\end{array}\right.
$$

where $\mathbf{v}_{\vartheta}^{m}$ is the virtual displacement field of the $\vartheta_{t h}$ representative material microstructure belonging to the kinematically admissible displacement space $\overline{\mathrm{U}}\left(\Omega_{\vartheta}^{m}\right)$.

\section{Sensitivity analysis for the dynamic multiscale design}

The dynamic multiscale topology optimization formulation can be directly solved by many well-established gradient-based algorithms [22,23]. Hence, sensitivity information of the objective and constraint functions with respect to the design variables are required. In the proposed formulation, there exist three kinds of the design variables, namely the element densities in the FMDO, the macro and micro expansion coefficients of the CSRBFs in the concurrent topology optimization.

\subsection{Sensitivity analysis of the FMDO}

In the FMDO, the first-order derivatives of the displacement response with respect to the element densities are computed based on the adjoint variable method [62], and the final form is given as:

$$
\frac{\partial \mathbf{U}}{\partial \rho_{e}}=-\left(\mathbf{K}_{d}\right)^{-1} \frac{\partial \mathbf{K}_{d}}{\partial \rho_{e}} \mathbf{U}
$$

According to the chain rule of the differential, the first-order derivatives of the objective with respect to the element densities are written as:

$$
\frac{\partial J}{\partial \rho_{e}}=\frac{\partial J_{R}}{\partial \rho_{e}}+i \frac{\partial J_{I}}{\partial \rho_{e}}=\mathbf{F}^{T} \frac{\partial \mathbf{U}}{\partial \rho_{e}}=-\mathbf{U}^{T} \frac{\partial \mathbf{K}_{d}}{\partial \rho_{e}} \mathbf{U}
$$


We can obtain the first-orders of the real and image of the dynamic compliance with respect to the design variables. The first-order derivative of the dynamic stiffness matrix can be derived by:

$$
\frac{\partial \mathbf{K}_{d}}{\partial \rho_{e}}=-\omega^{2} \frac{\partial \mathbf{M}}{\partial \rho_{e}}+i \omega \frac{\partial \mathbf{C}}{\partial \rho_{e}}+\frac{\partial \mathbf{K}}{\partial \rho_{e}}
$$

where

$$
\left\{\begin{array}{l}
\frac{\partial \mathbf{K}}{\partial \rho_{e}}=\mathbf{K}_{0} ; \frac{\partial \mathbf{M}}{\partial \rho_{e}}=\mathbf{M}_{0} \\
\frac{\partial \mathbf{C}}{\partial \rho_{e}}=\mathcal{A} \frac{\partial \mathbf{M}}{\partial \rho_{e}}+\mathcal{B} \frac{\partial \mathbf{K}}{\partial \rho_{e}}
\end{array}\right.
$$

where $\mathbf{K}_{0}$ and $\mathbf{M}_{0}$ are the stiffness and mass matrices of the solid element, respectively. In Eq. (13), the first-order derivatives of the objective function can be derived, given by:

$$
\frac{\partial J_{d}}{\partial \rho_{e}}=\frac{1}{J_{d}}\left(J_{R} \frac{\partial J_{R}}{\partial \rho_{e}}+J_{I} \frac{\partial J_{I}}{\partial \rho_{e}}\right)
$$

The derivatives of the objective function with respect to the element densities can be derived by Eqs. (23), (25) and (26). Meanwhile, the derivative of the volume constraint is given as:

$$
\frac{\partial G_{d}}{\partial \rho_{e}}=\partial\left(\sum_{e=1}^{N_{e}} \rho_{e} v_{0}-V_{d}\right) / \partial \rho_{e}=v_{0}
$$

\subsection{Macro sensitivity analysis in the concurrent topology optimization}

The macro expansion coefficients of the CSRBFs are the design variables in the macro optimization. In the LSM, the material derivative [63] is employed to calculate the derivatives of the objective and constraint functions with respect to the macro design variables. Firstly, the dynamic compliance can be transformed a form based on the macro elastic equilibrium state equation, given as:

$$
J(\mathbf{u}, \Phi)=\sum_{\vartheta=1}^{\Theta} \int_{D^{M}}\left\{(1+i \omega \mathcal{B}) \boldsymbol{\varepsilon}\left(\mathbf{u}_{\vartheta}^{M}\right) \mathbf{E}_{\vartheta}^{H} \boldsymbol{\varepsilon}\left(\mathbf{u}_{\vartheta}^{M}\right)+\left(i \omega \mathcal{A}-\omega^{2}\right) \psi_{\vartheta}^{H} \mathbf{u}_{\vartheta}^{M} \mathbf{u}_{\vartheta}^{M}\right\} H\left(\Phi_{\vartheta}^{M}\right) d \Omega_{\vartheta}^{M}
$$

The material derivative of the structural dynamic compliance is derived as:

$$
\dot{j}=\sum_{\vartheta=1}^{\Theta}\left\{\begin{array}{l}
2 \int_{D^{M}}\left\{(1+i \omega \mathcal{B}) \boldsymbol{\varepsilon}\left(\dot{\mathbf{u}}_{\vartheta}^{M}\right) \mathbf{E}_{\vartheta}^{H} \boldsymbol{\varepsilon}\left(\mathbf{u}_{\vartheta}^{M}\right)+\left(i \omega \mathcal{A}-\omega^{2}\right) \psi_{\vartheta}^{H} \dot{\mathbf{u}}_{\vartheta}^{M} \mathbf{u}_{\vartheta}^{M}\right\} H\left(\Phi_{\vartheta}^{M}\right) d \Omega_{\vartheta}^{M} \\
+\int_{\Gamma^{M}}\left\{(1+i \omega \mathcal{B}) \boldsymbol{\varepsilon}\left(\mathbf{u}_{\vartheta}^{M}\right) \mathbf{E}_{\vartheta}^{H} \boldsymbol{\varepsilon}\left(\mathbf{u}_{\vartheta}^{M}\right)+\left(i \omega \mathcal{A}-\omega^{2}\right) \psi_{\vartheta}^{H} \mathbf{u}_{\vartheta}^{M} \mathbf{u}_{\vartheta}^{M}\right\} v_{\mathbf{n}}^{M} d \Gamma^{M}
\end{array}\right\}
$$

Taking the material derivative on both sides of the state equation, the following equation is obtained as: 


$$
\begin{aligned}
& \sum_{\vartheta=1}^{\Theta}\left\{\begin{array}{l}
(1+i \omega \mathcal{B})\left[k\left(\dot{\mathbf{u}}_{\vartheta}^{M}, \mathbf{v}_{\vartheta}^{M}\right)+k\left(\mathbf{u}_{\vartheta}^{M}, \dot{\mathbf{v}}_{\vartheta}^{M}\right)+\int_{\Gamma^{M}} \boldsymbol{\varepsilon}\left(\mathbf{u}_{\vartheta}^{M}\right) \mathbf{E}_{\vartheta}^{H} \boldsymbol{\varepsilon}\left(\mathbf{v}_{\vartheta}^{M}\right) v_{\mathbf{n}}^{M} d \Gamma^{M}\right] \\
+\left(i \omega \mathcal{A}-\omega^{2}\right)\left[m\left(\dot{\mathbf{u}}_{\vartheta}^{M}, \mathbf{v}_{\vartheta}^{M}\right)+m\left(\mathbf{u}_{\vartheta}^{M}, \dot{\mathbf{v}}_{\vartheta}^{M}\right)+\int_{\Gamma^{M}} \psi_{\vartheta}^{H} \mathbf{u}_{\vartheta}^{M} \mathbf{v}_{\vartheta}^{M} v_{\mathbf{n}}^{M} d \Gamma^{M}\right]
\end{array}\right\} \\
& =\left\{\begin{array}{l}
\int_{D^{M}} p \dot{\mathbf{v}}^{M} H\left(\Phi^{M}\right) d \Omega^{M}+\int_{\Gamma^{M}} p \mathbf{v}^{M} v_{\mathbf{n}}^{M} d \Gamma^{M}+\cdots \\
\int_{\Gamma^{M}} f \dot{\mathbf{v}}^{M} d \Gamma^{M}+\int_{\Gamma^{M}}\left[\nabla\left(f \mathbf{v}^{M}\right) \cdot \mathbf{n}+\kappa\left(f \mathbf{v}^{M}\right)\right] v_{\mathbf{n}}^{M} d \Gamma^{M}
\end{array}\right\}
\end{aligned}
$$

where $\dot{\mathbf{u}}_{\vartheta}^{M}$ and $\dot{\mathbf{v}}_{\vartheta}^{M}$ are the partial derivatives of $\mathbf{u}_{\vartheta}^{M}$ and $\mathbf{v}_{\vartheta}^{M}$ with respect to the time variable respectively. Considering that $\dot{\mathbf{v}}_{\vartheta}^{M} \in \overline{\mathrm{U}}\left(\Omega^{M}\right)$, we would gain the following vibrational state equation, as:

$$
\sum_{\vartheta=1}^{\Theta}\left[(1+i \omega \mathcal{B}) k\left(\mathbf{u}_{\vartheta}^{M}, \dot{\mathbf{v}}_{\vartheta}^{M}\right)+\left(i \omega \mathcal{A}-\omega^{2}\right) m\left(\mathbf{u}_{\vartheta}^{M}, \dot{\mathbf{v}}_{\vartheta}^{M}\right)\right]=\left\{\int_{D^{M}} p \dot{\mathbf{v}}^{M} H\left(\Phi^{M}\right) d \Omega^{M}+\int_{\Gamma^{M}} f \dot{\mathbf{v}}^{M} d \Gamma^{M}\right\}
$$

Substituting Eq. (31) into Eq. (30) and eliminating all the terms that contains $\dot{\mathbf{v}}_{\vartheta}^{M}$, it yields:

$$
\begin{aligned}
& \sum_{\vartheta=1}^{\Theta}\left[(1+i \omega \mathcal{B}) k\left(\dot{\mathbf{u}}_{\vartheta}^{M}, \mathbf{v}_{\vartheta}^{M}\right)+\left(i \omega \mathcal{A}-\omega^{2}\right) m\left(\dot{\mathbf{u}}_{\vartheta}^{M}, \mathbf{v}_{\vartheta}^{M}\right)\right] \\
& =\int_{\Gamma^{M}}\left\{\begin{array}{l}
-\sum_{\vartheta=1}^{\Theta}\left[(1+i \omega \mathcal{B}) \varepsilon\left(\mathbf{u}_{\vartheta}^{M}\right) \mathbf{E}_{\vartheta}^{H} \boldsymbol{\varepsilon}\left(\mathbf{v}_{\vartheta}^{M}\right)+\left(i \omega \mathcal{A}-\omega^{2}\right) \psi_{\vartheta}^{H} \mathbf{u}_{\vartheta}^{M} \mathbf{v}_{\vartheta}^{M}\right] \\
+\left\{p \mathbf{v}^{M}+\left[\nabla\left(f \mathbf{v}^{M}\right) \cdot \mathbf{n}+\kappa\left(f \mathbf{v}^{M}\right)\right]\right\}
\end{array}\right\} v_{\mathbf{n}}^{M} d \Gamma^{M}
\end{aligned}
$$

Because the problem for the dynamic compliance is the self-adjoint [52-54], the material derivative of the structural dynamic compliance can be transformed into a new form, defined as:

$$
\dot{j}(\mathbf{u}, \Phi)=\int_{\Gamma^{M}} \boldsymbol{r}(\mathbf{u}, \Phi) v_{\mathbf{n}}^{M} d \Gamma^{M}
$$

where

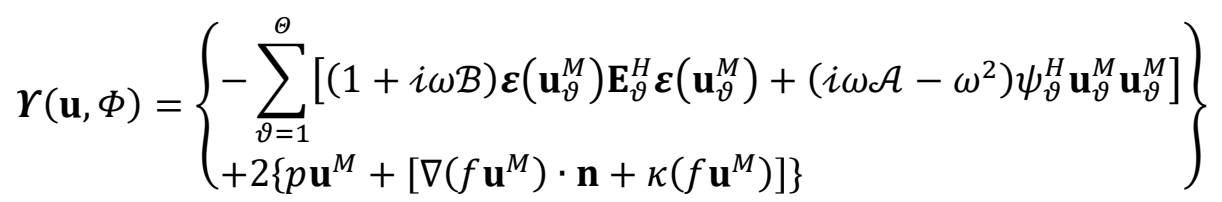

The Lagrange function $L$ for the concurrent topology optimization is defined by:

$$
L=J+(1+i \omega \mathcal{B}) k+\left(i \omega \mathcal{A}-\omega^{2}\right) m-l+\lambda G_{M}
$$

where $\lambda$ is the Lagrange multiplier, and the derivative of the Lagrange function $L$ is derived as:

$$
\frac{d L}{d t}=\int_{\Gamma^{M}} \boldsymbol{\beta}(\mathbf{u}, \Phi) v_{\mathbf{n}}^{M} d \Gamma^{M}
$$

where 


$$
\boldsymbol{\beta}(\mathbf{u}, \Phi)=\boldsymbol{Y}(\mathbf{u}, \Phi)+\lambda \sum_{\vartheta=1}^{\Theta}\left[\int_{D_{\vartheta}^{m}} H\left(\Phi_{\vartheta}^{m}\right) d \Omega_{\vartheta}^{m}\right]
$$

and $\kappa$ is the mean curvature in two dimensions. Now, recalling the normal velocity field $v_{\mathbf{n}}^{M}$ in Eq. (12) and substituting it into Eq. (36), the derivative of the Lagrange function is further written as:

$$
\frac{d L}{d t}=\int_{\Gamma^{M}}\left\{\boldsymbol{\beta}(\mathbf{u}, \Phi) \frac{\boldsymbol{\varphi}^{T}(\mathbf{x})}{\left|\nabla \boldsymbol{\varphi}^{T}(\mathbf{x}) \boldsymbol{\alpha}^{M}(t)\right|}\right\} \frac{d \boldsymbol{\alpha}^{M}(t)}{d t} d \Gamma^{M}
$$

Eq. (38) can be expanded as a new form:

$$
\frac{d L}{d t}=\mathcal{P}^{T} \frac{d \boldsymbol{\alpha}^{M}(t)}{d t}+\mathcal{Q}^{T} \frac{d \boldsymbol{\alpha}^{M}(t)}{d t}
$$

where $\mathcal{P}^{T}$ and $\mathcal{Q}^{T}$ are defined as:

$$
\left\{\begin{array}{l}
\mathcal{P}^{T}=\int_{\Gamma^{M}} \boldsymbol{r}(\mathbf{u}, \Phi) \frac{\boldsymbol{\varphi}^{T}(\mathbf{x})}{\left|\nabla \boldsymbol{\varphi}^{T}(\mathbf{x}) \boldsymbol{\alpha}^{M}(t)\right|} d \Gamma^{M} \\
\mathcal{Q}^{T}=\left\{\lambda \sum_{\vartheta=1}^{\Theta}\left[\int_{D_{\vartheta}^{m}} H\left(\Phi_{\vartheta}^{m}\right) d \Omega_{\vartheta}^{m}\right]\right\} \int_{\Gamma^{M}} \frac{\boldsymbol{\varphi}^{T}(\mathbf{x})}{\left|\nabla \boldsymbol{\varphi}^{T}(\mathbf{x}) \boldsymbol{\alpha}^{M}(t)\right|} d \Gamma^{M}
\end{array}\right.
$$

On the other hand, the derivative of the Lagrange function $L$ can be given based on the chain rule, as:

$$
\frac{d L}{d t}=\left(\frac{\partial J}{\partial \boldsymbol{\alpha}^{M}}+\lambda \frac{\partial G_{M}}{\partial \boldsymbol{\alpha}^{M}}\right) \frac{d \boldsymbol{\alpha}^{M}(t)}{d t}
$$

Thus, the first-order derivatives of the dynamic compliance and the constraint function can be obtained by comparing the corresponding terms in Eqs. (39) and (41), given as:

$$
\left\{\begin{array}{l}
\frac{\partial J}{\partial \boldsymbol{\alpha}^{M}}=\int_{\Gamma^{M}} \boldsymbol{r}(\mathbf{u}, \Phi) \frac{\boldsymbol{\varphi}^{T}(\mathbf{x})}{\left|\nabla \boldsymbol{\varphi}^{T}(\mathbf{x}) \boldsymbol{\alpha}^{M}(t)\right|} d \Gamma^{M} \\
\frac{\partial G_{M}}{\partial \boldsymbol{\alpha}^{M}}=\left\{\sum_{\vartheta=1}^{\Theta}\left[\int_{D_{\vartheta}^{m}} H\left(\Phi_{\vartheta}^{m}\right) d \Omega_{\vartheta}^{m}\right]\right\} \int_{\Gamma^{M}} \frac{\boldsymbol{\varphi}^{T}(\mathbf{x})}{\left|\nabla \boldsymbol{\varphi}^{T}(\mathbf{x}) \boldsymbol{\alpha}^{M}(t)\right|} d \Gamma^{M}
\end{array}\right.
$$

In order to improve the numerical efficiency, the design sensitivities expressed by the boundary integration scheme can be transformed into the following volume integration [16,17,24,29].

$$
\left\{\begin{array}{l}
\frac{\partial J}{\partial \boldsymbol{\alpha}^{M}}=\int_{\mathrm{D}^{M}} \boldsymbol{r}(\mathbf{u}, \Phi) \boldsymbol{\varphi}^{T}(\mathbf{x}) \delta\left(\Phi^{M}\right) d \Omega^{M} \\
\frac{\partial G_{M}}{\partial \boldsymbol{\alpha}^{M}}=\left\{\sum_{\vartheta=1}^{\Theta}\left[\int_{D_{\vartheta}^{m}} H\left(\Phi_{\vartheta}^{m}\right) d \Omega_{\vartheta}^{m}\right]\right\} \int_{\mathrm{D}^{M}} \boldsymbol{\varphi}^{T}(\mathbf{x}) \delta\left(\Phi^{M}\right) d \Omega^{M}
\end{array}\right.
$$

According to the sensitivity analysis in the FMDO defined by Eqs. (23) and (26), the first-order derivatives of the objective function in the concurrent topology optimization can be calculated, as: 


$$
\frac{\partial J_{d}}{\partial \boldsymbol{\alpha}^{M}}=\frac{1}{J_{d}}\left(J_{R} \frac{\partial J_{R}}{\partial \boldsymbol{\alpha}^{M}}+J_{I} \frac{\partial J_{I}}{\partial \boldsymbol{\alpha}^{M}}\right)
$$

where

$$
\frac{\partial J}{\partial \boldsymbol{\alpha}^{M}}=\frac{\partial J_{R}}{\partial \boldsymbol{\alpha}^{M}}+i \frac{\partial J_{I}}{\partial \boldsymbol{\alpha}^{M}}=\int_{\mathrm{D}^{M}} \boldsymbol{r}(\mathbf{u}, \Phi) \boldsymbol{\varphi}^{T}(\mathbf{x}) \delta\left(\Phi^{M}\right) d \Omega^{M}
$$

\subsection{Micro sensitivity analysis in the concurrent topology optimization}

The derivatives of the dynamic compliance with respect to the micro design variables are computed based on the chain rule, given as:

$$
\begin{aligned}
& \frac{\partial J}{\partial \boldsymbol{\alpha}_{\vartheta}^{m}}=\frac{\partial J_{R}}{\partial \boldsymbol{\alpha}_{\vartheta}^{m}}+i \frac{\partial J_{I}}{\partial \boldsymbol{\alpha}_{\vartheta}^{m}}=\cdots \\
& \int_{D^{M}}\left\{(1+i \omega \mathcal{B}) \boldsymbol{\varepsilon}\left(\mathbf{u}_{\vartheta}^{M}\right) \frac{\partial \mathbf{E}_{\vartheta}^{H}}{\partial \boldsymbol{\alpha}_{\vartheta}^{m}} \boldsymbol{\varepsilon}\left(\mathbf{u}_{\vartheta}^{M}\right)+\left(i \omega \mathcal{A}-\omega^{2}\right) \frac{\partial \psi_{\vartheta}^{H}}{\partial \boldsymbol{\alpha}_{\vartheta}^{m}} \mathbf{u}_{\vartheta}^{M} \mathbf{u}_{\vartheta}^{M}\right\} H\left(\Phi_{\vartheta}^{M}\right) d \Omega_{\vartheta}^{M}
\end{aligned}
$$

It can be found that the key to solve the sensitivities from Eq. (46) lies in the derivatives of $\mathbf{E}_{\vartheta}^{H}$ and $\psi_{\vartheta}^{H}$ with respect to the micro design variables. The first-order derivative of the homogenized elastic tensor $\mathbf{E}_{\vartheta}^{H}$ with respect to time $t$ is derived by:

$$
\frac{\partial E_{\vartheta(i j k l)}^{H}}{\partial t}=\frac{1}{\left|\Omega_{\vartheta}^{m}\right|} \int_{D_{\vartheta}^{m}}\left(\varepsilon_{p q}^{0(i j)}-\varepsilon_{p q}^{*}\left(u_{\vartheta}^{m(i j)}\right)\right) E_{p q r s}\left(\varepsilon_{r s}^{0(k l)}-\varepsilon_{r s}^{*}\left(u_{\vartheta}^{m(k l)}\right)\right) v_{\vartheta, \mathbf{n}}^{m}\left|\nabla \Phi_{\vartheta}^{m}\right| \delta\left(\Phi_{\vartheta}^{m}\right) d \Omega_{\vartheta}^{m}
$$

Recalling the normal velocity field $v_{\vartheta, \mathbf{n}}^{m}$ of the representative microstructure in Eq. (12), and substituting it into Eq. (47), yields:

$$
\frac{\partial E_{\vartheta}^{H}(i j k l)}{\partial t}=\frac{1}{\left|\Omega_{\vartheta}^{m}\right|} \int_{D_{\vartheta}^{m}}\left(\varepsilon_{p q}^{0(i j)}-\varepsilon_{p q}^{*}\left(u_{\vartheta}^{m(i j)}\right)\right) E_{p q r s}\left(\varepsilon_{r s}^{0(k l)}-\varepsilon_{r s}^{*}\left(u_{\vartheta}^{m(k l)}\right)\right) \boldsymbol{\varphi}^{T}(\mathbf{y}) \delta\left(\Phi_{\vartheta}^{m}\right) d \Omega_{\vartheta}^{m} \frac{d \boldsymbol{\alpha}_{\vartheta}^{m}}{d t}
$$

On the other side, based on the chain rule, the first-order derivative of $\mathbf{E}_{\vartheta}^{H}$ with respect to time $t$ is given:

$$
\frac{\partial E_{\vartheta(i j k l)}^{H}}{\partial t}=\frac{\partial E_{\vartheta(i j k l)}^{H}}{\partial \boldsymbol{\alpha}_{\vartheta}^{m}} \frac{d \boldsymbol{\alpha}_{\vartheta}^{m}}{d t}
$$

According to the Eqs. (48) and (49), the first-order derivatives of $\mathbf{E}_{\vartheta}^{H}$ with respect to the micro expansion coefficients can be given as:

$$
\frac{\partial E_{\vartheta(i j k l)}^{H}}{\partial \boldsymbol{\alpha}_{\vartheta}^{m}}=\frac{1}{\left|\Omega_{\vartheta}^{m}\right|} \int_{D_{\vartheta}^{m}}\left(\varepsilon_{p q}^{0(i j)}-\varepsilon_{p q}^{*}\left(u_{\vartheta}^{m(i j)}\right)\right) E_{p q r s}\left(\varepsilon_{r s}^{0(k l)}-\varepsilon_{r s}^{*}\left(u_{\vartheta}^{m(k l)}\right)\right) \boldsymbol{\varphi}^{T}(\mathbf{y}) \delta\left(\Phi_{\vartheta}^{m}\right) d \Omega_{\vartheta}^{m}
$$


Similarly, the derivatives of the homogenized mass density $\psi_{\vartheta}^{H}$ with respect to the micro design variables can be stated as:

$$
\frac{\partial \psi_{\vartheta}^{H}}{\partial \boldsymbol{\alpha}_{\vartheta}^{m}}=\frac{1}{\left|\Omega_{\vartheta}^{m}\right|} \int_{D_{\vartheta}^{m}} \psi_{0} \boldsymbol{\varphi}^{T}(\mathbf{y}) \delta\left(\Phi_{\vartheta}^{m}\right) d \Omega_{\vartheta}^{m}
$$

Finally, the first-order derivatives of the dynamic compliance $J$ with respect to the micro design variables are attained by substituting Eqs. (50) and (51) into Eq. (46). The derivatives of the objective function $J_{d}$ with respect to the design variables can be computed by the chain rule, as follows:

$$
\frac{\partial J_{d}}{\partial \boldsymbol{\alpha}_{\vartheta}^{m}}=\frac{1}{J_{d}}\left(J_{R} \frac{\partial J_{R}}{\partial \boldsymbol{\alpha}_{\vartheta}^{m}}+J_{I} \frac{\partial J_{I}}{\partial \boldsymbol{\alpha}_{\vartheta}^{m}}\right)
$$

Similarly, the first-order derivative of the micro volume constraint $G_{\vartheta}^{m}$ is defined by:

$$
\frac{\partial G_{\vartheta}^{m}}{\partial \boldsymbol{\alpha}_{\vartheta}^{m}}=\int_{D_{\vartheta}^{m}} \boldsymbol{\varphi}^{T}(\mathbf{y}) \delta\left(\Phi_{\vartheta}^{m}\right) d \Omega_{\vartheta}^{m}
$$

\section{Numerical Implementations}

\subsection{Quasi-static Ritz vector (QSRV) method}

Usually, the dynamic topology optimization is computationally expensive due to a large number of degrees of freedom. Hence, many model reduction schemes, like the mode superposition method [5], the Ritz vector (RV) method [55] and the Quasi-static Ritz vector (QSRV) method [55], are required. Here, we adopt the QSRV method to approximate the dynamic responses since it can guarantee the numerical accuracy while reduces the computational demands. In the QSRV, the reduction bases $\Psi$ are constructed considering the external force $\mathbf{F}$, the excitation frequency (acting as the center frequency $\omega$ ), the mass matrix $\mathbf{M}$ and the stiffness matrix $\mathbf{K}$. The first basis $\zeta_{1}$ is developed by solving the dynamic equation and normalizing the dynamic displacement to the mass matrix, given as:

$$
\zeta_{1}=\frac{1}{\left(\zeta_{1}^{*}\right)^{T} \mathbf{M} \zeta_{1}^{*}} \zeta_{1}^{*}
$$

where

$$
\zeta_{1}^{*}=\left(\mathbf{K}-\omega^{2} \mathbf{M}\right)^{-1} \mathbf{F}
$$

The rest bases are sequentially constructed by considering the previously calculated bases, center frequency $\omega$, mass matrix $\mathbf{M}$ and stiffness matrix $\mathbf{K}$, defined by:

$$
\zeta_{j}^{*}=\left(\mathbf{K}-\omega^{2} \mathbf{M}\right)^{-1}\left(\mathbf{M} \zeta_{j-1}\right)
$$


By orthogonalizing:

$$
\zeta_{j}^{* *}=\zeta_{j}^{*}-\sum_{k=1}^{j-1}\left(\left(\zeta_{k}^{*}\right)^{T} \mathbf{M} \zeta_{k}^{*}\right) \zeta_{k}
$$

By normalizing:

$$
\zeta_{j}=\frac{1}{\left(\zeta_{j}^{* *}\right)^{T} \mathbf{M} \zeta_{j}^{* *}} \zeta_{j}^{* *}
$$

The reduction bases are utilized to approximate dynamic response, which can transform a large number of system equations into a small number of equations. The approximated response can be written as:

$$
\mathbf{U} \approx \mathbf{U}_{r}=\Psi Q
$$

where

$$
\left\{\begin{array}{l}
\mathbf{U}_{r}=\left\{\zeta_{1}, \zeta_{2}, \cdots, \zeta_{j}, \cdots, \zeta_{n_{r}}\right\} \quad\left(n_{r} \leq n\right) \\
\left\{\Psi^{T}\left(-\omega^{2} \mathbf{M}+i \omega \mathbf{C}+\mathbf{K}\right) \Psi\right\} Q=\Psi^{T} \mathbf{F}
\end{array}\right.
$$

where $\mathbf{U}_{r}$ denotes the approximated displacement responses.

\subsection{Connectivity mechanism}

In the multiscale design with multiple material microstructures, the connectivity in adjacent microstructures is a critical issue in numerical implementation $[26,35,46,48]$. As discussed in [46], the connectivity should be kept well in nature in the multiscale design of cellular composites, due to the basic fact that a continuous loading transmission path should be occurred in the design domain. Meanwhile, a kinematically connective constraint approach $[26,35,48]$ is developed to guarantee the connectivity between the adjacent material microstructures. In this work, the latter scheme is employed, a number of the connectors serving as the nondesign components are pre-defined within material microstructures, as clearly illustrated in Fig. 4, namely four connectors in 2D unit cells and eight connectors in 3D microstructures.
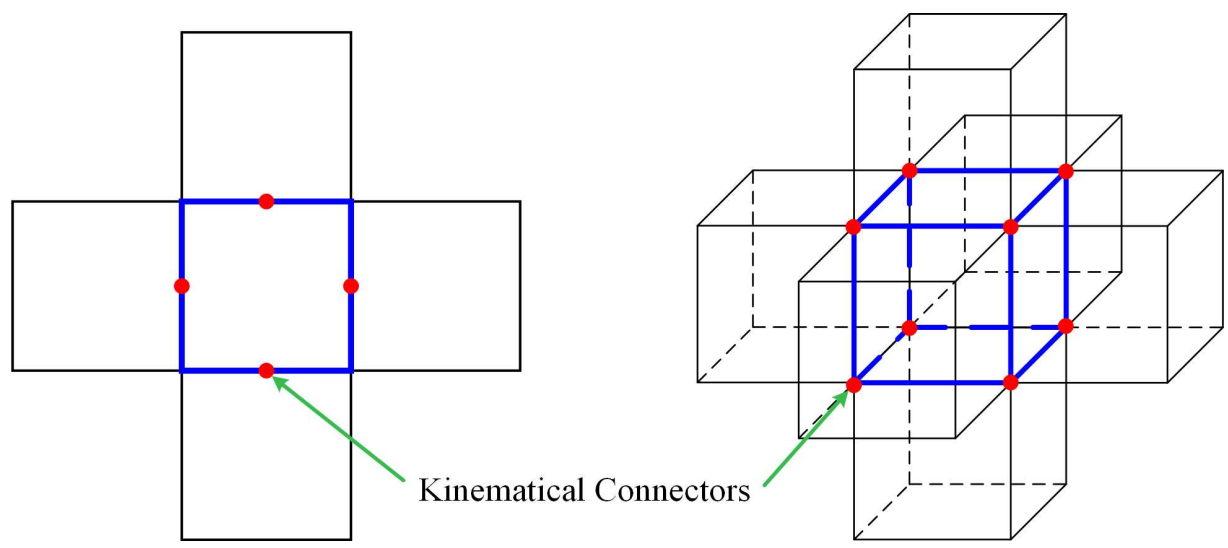

Fig. 4. Predefined connectors (red dots) between adjacent microstructures in $2 \mathrm{D}$ and $3 \mathrm{D}$ 


\subsection{Updating scheme for design variables}

In this work, the OC algorithm is employed to update the design variables at two scales, owing to its superior features for problems with a large number of design variables but only with a single constraint [22]. Firstly, a heuristic updating scheme is established for the design variables in the FMDO, given as:

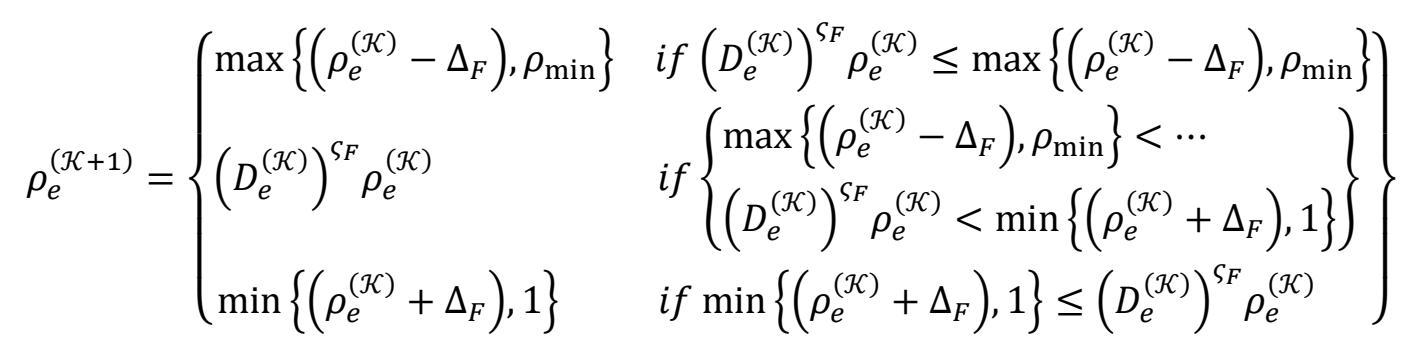

where $\mathcal{K}$ denote the current iteration step. $\Delta_{F}$ and $\varsigma_{F}$ are the move limit and damping coefficient for the design variables in the FMDO, respectively. $D_{e}^{(\mathcal{K})}$ is the corresponding updating factor, given by:

$$
D_{e}^{(\mathcal{K})}=-\left[\frac{\partial J_{d}}{\partial \rho_{e}^{(\mathcal{K})}} / \max \left(\mu, \Lambda_{F} \frac{\partial G_{d}}{\partial \rho_{e}^{(\mathcal{K})}}\right)\right]
$$

where $\mu$ is a very small positive constant to avoid the denominator equal to 0 , and $\Lambda_{F}$ is the Lagrange multiplier in the FMDO. The bi-sectioning algorithm [8] is an efficient and simple scheme to calculate the Lagrange multiplier, where a lower and an upper bounds are repeatedly evaluated by pushing the volume back to satisfy the volume constraint. The details are given as follows:

Step 1: Set lower bound $\Lambda_{F}^{\min }$ and upper bound $\Lambda_{F}^{\max }$ of the Lagrange multiplier $\Lambda_{F}$.

Step 2: Let $\Lambda_{F}^{\text {temp }}=\left(\Lambda_{F}^{\min }+\Lambda_{F}^{\max }\right) / 2$.

Step 3: Set $\Lambda_{F}=\Lambda_{F}^{\text {temp }}$, and update the design variables by Eq. (61).

Step 4: Calculate the volume constraint $G_{d}$.

Step 5: Check $G_{d}=0$ ? if yes, stop and return the design variables. If no, go to Step 6.

Step 6: If $G_{d}>0$, set $\Lambda_{F}^{\min } \leftarrow \Lambda_{F}^{\text {temp }}$; Else if $G_{d}<0$, set $\Lambda_{F}^{\max } \leftarrow \Lambda_{F}^{\text {temp }}$.

Step 7: Go to Step 2 and repeat until $G_{d}=0$.

In the concurrent topology optimization for the macrostructure and multiple microstructures, the two-scale design variables are contained, namely the macro and micro expansion coefficients. As already pointed out in several works $[16,17,24,29,49,54]$, one of the most important contribution of the PLSM is the elimination of the limitation of the signed distance function, and which allow for a free surface evolution driven by the sensitivity information. On the other side, the interpolated coefficients for the LSFs, namely the macro and micro expansion coefficients in the concurrent topology optimization, working as the design variables do 
not have the definite bounds in the updating by the sensitivity analysis, which might affect the numerical stability to some extent in the optimization [64]. In the current work, we introduce a normalization scheme for the two-scale design variables to force them into a lateral bound $[0,1]$.

\subsubsection{The normalization of the two-scale design variables}

The maximum and minimum values of the initial LSFs at two scales are symbolled by $\Phi_{\min }^{M}$ and $\Phi_{\max }^{M}$, $\Phi_{\vartheta, \text { min }}^{m}$ and $\Phi_{\vartheta, \text { max }}^{m}$, respectively. Based on Eq. (9), the lower and upper bounds for the initial values of the macro and micro expansion coefficients can be calculated, denoted by $\alpha_{\min }^{M}$ and $\alpha_{\max }^{M}, \alpha_{\vartheta, \min }^{m}$ and $\alpha_{\vartheta, \max }^{m}$, respectively. The normalization for the two-scale design variables is defined by:

$$
\left\{\begin{array}{l}
\bar{\alpha}_{i}^{M}=\frac{\alpha_{i}^{M}-\alpha_{\min }^{M}}{\alpha_{\max }^{M}-\alpha_{\min }^{M}} \\
\bar{\alpha}_{\vartheta, j}^{m}=\frac{\alpha_{\vartheta, j}^{m}-\alpha_{\vartheta, \min }^{m}}{\alpha_{\vartheta, \max }^{m}-\alpha_{\vartheta, \min }^{m}}
\end{array}\right.
$$

We can easily see that all the normalized design variables will be evolved within the bound $[0,1]$. After updating the normalized design variables, the actual design variables can be obtained by:

$$
\left\{\begin{array}{l}
\alpha_{i}^{M}=\bar{\alpha}_{i}^{M} \times\left(\alpha_{\max }^{M}-\alpha_{\min }^{M}\right)+\alpha_{\min }^{M} \\
\alpha_{\vartheta, j}^{m}=\bar{\alpha}_{\vartheta, j}^{m} \times\left(\alpha_{\vartheta, \max }^{m}-\alpha_{\vartheta, \min }^{m}\right)+\alpha_{\vartheta, \min }^{m}
\end{array}\right.
$$

Hence, the updating of the macro and micro expansion coefficients is kept in the ranges $\alpha_{\min }^{M}$ and $\alpha_{\max }^{M}$, $\alpha_{\vartheta \text {,min }}^{m}$ and $\alpha_{\vartheta \text {,max }}^{m}$, respectively, which can avoid the high fluctuations of the LSFs during the optimization. The normalized design variables at two scales are also updated by the OC algorithm, and the bi-sectioning algorithm is also applied to evolve the Lagrange multipliers at two scales for the volume constraints in the macro and micro designs. The details are shown in Section 6.3. The only difference is that the move limit and damping factor should be chosen appropriately for the concurrent topology optimization.

\section{Numerical Examples}

In this section, 2D and 3D numerical examples are demonstrated to display the effectiveness of the dynamic multiscale topology optimization for cellular composites. In the homogenization [61], noting that material microstructures have no specific sizes but should ensure the periodicity condition within the macrostructure. For numerical simplicity, the sizes of material microstructures in all normal directions are defined to be 1 $\mathrm{mm}$. The material has the Young's modulus $210 \mathrm{GPa}$, Possion's ratio 0.3 and mass density $7800 \mathrm{~kg} / \mathrm{m}^{3}$. The coefficients are defined as: $\mathcal{A}=0.03$ and $\mathcal{B}=0.001$, respectively. The damping coefficients and move limits for three kinds of the design variables are defined as: 0.5 and $0.2,0.5$ and $0.01,0.5$ and 0.002 , 
respectively. The termination criterion for the FMDO is that the difference of the design variables between two successive iterations is less than 0.001 , and the concurrent topology optimization will terminate if the difference of the objective function between two adjacent steps is less than 1e-4 or the maximum 200 steps are reached.

\subsection{Clamped beam}

In Fig. 5, a clamped beam is fixed at both sides and loaded with a downward excitation force with a certain frequency $\omega=80 \mathrm{~Hz}$, namely $\mathbf{F}=-1 e 6 e^{i 80 t}$. The design domain has the length $L=1.05 \mathrm{~m}$, the height $H=0.15 \mathrm{~m}$, which is discretized with $210 \times 30$ four-node finite elements. The discretization of all material microstructures uses a mesh of $30 \times 30$ finite elements. The maximum of the global volume fraction $V_{M}$ is set to be $30 \%$, and the volume fraction $V_{d}$ in the FDMO is set as $45 \%$.

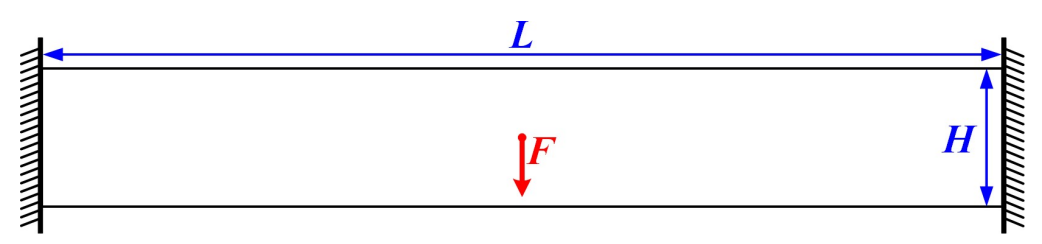

Fig. 5. The clamped beam

\subsubsection{Dynamic multiscale topology optimization design}

\section{(a) Stage 1: FMDO}

As shown in Fig. 6 (a), the continuous distribution of the element densities are obtained by the formulation in Eq. (13). It can be easily found that a huge number of the intermediate element densities are occurred in the design, and the number of the representative microstructures to be optimized is considerably increased. The scheme 1 (S1) of the regularization mechanism, defined in Table 1, is applied to process the element densities, and the regularized distribution of the densities is shown in Fig. $6(b)$. As we can see, the discrete element densities with only a limited number (5) are distributed in the multi-regional manner, and each of them is uniformly filled within the corresponding sub area of the macrostructure. The macro design domain is divided into five different sub regions plotted by different colors, namely the white, blue, green, red and black. It should be noted that the regularized element densities are equal to zero (plotted by the white) if they are between 0.00 and 0.20 and the regularized element densities are equal to unit (plotted by the black) if they belong to the last group $(0.8-1.0)$ to stable the numerical optimization.

Table 1. Scheme 1 (S1) of the regularization mechanism

\begin{tabular}{|c|c|c|c|c|c|}
\hline \multirow{3}{*}{$\begin{array}{c}\text { Scheme } \\
\text { S1 }\end{array}$} & \multicolumn{5}{|c|}{ The defined thresholds in different groups } \\
\hline & Group 1 & Group 2 & Group 3 & Group 4 & Group 5 \\
\hline & {$\left[\begin{array}{ll}0 & 0.2\end{array}\right]$} & {$\left[\begin{array}{lll}0.2 & 0.4)\end{array}\right.$} & {$\left[\begin{array}{lll}0.4 & 0.6\end{array}\right)$} & {$\left[\begin{array}{lll}0.6 & 0.8\end{array}\right)$} & {$\left[\begin{array}{ll}0.8 & 1\end{array}\right]$} \\
\hline
\end{tabular}




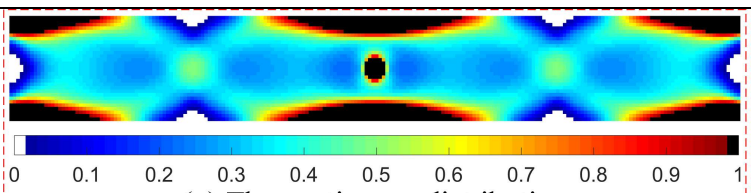

(a) The continuous distribution

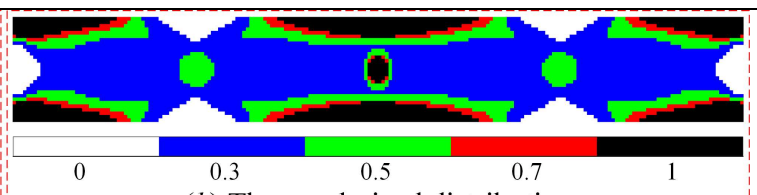

(b) The regularized distribution

Fig. 6. Two distributions in the FMDO

\section{(b) Stage 2: Concurrent topology optimization}

The regularized densities are chosen as the maximum value of the volume constraints for the representative microstructures, as shown in the micro volume constraints $G_{\vartheta}^{m}$. The topologies of both the macrostructure and the representative microstructures are concurrently optimized, subject to the regularized distribution of the densities shown in Fig. $6(b)$. The macro topology is optimized under the global volume constraint $G^{M}$ and the micro topologies are evolved subject to the local volume constraints $G_{\vartheta}^{m}$. The initial design of the macrostructure is defined in Fig. 7 (a), and the optimizations of material microstructures employ the same initial design displayed in Fig. 7 (b). The optimized topology of the macrostructure is indicated in Fig. 8, and the optimized results of four distinct microstructures (excluding void microstructure) are listed in Table 2, including the topologies, the $5 \times 5$ repetitive microstructures and the homogenized elastic tensors.

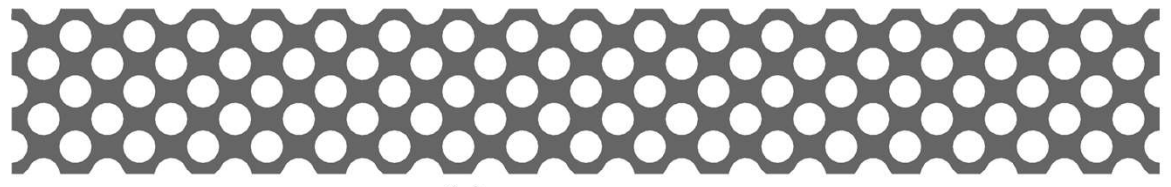

(a) macrostructure

Fig. 7. Initial designs at two scales

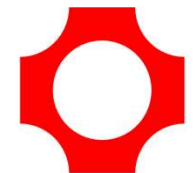

(b) material cell

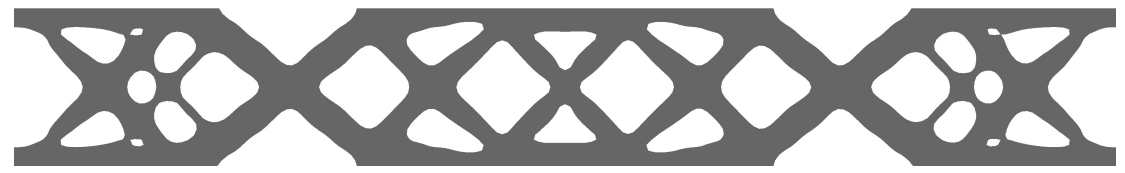

Fig. 8. The optimized topology of the macrostructure

Table 2. The optimized results of material microstructures

\begin{tabular}{|c|c|c|c|c|c|c|c|}
\hline$\vartheta$ & $\bar{\rho}_{\vartheta}$ & Material microstructure & $5 \times 5$ microstructures & & $\mathbf{E}_{\vartheta}^{H}$ & & \\
\hline 2 & 0.3 & & & {$\left[\begin{array}{c}2.23 \\
1.80 \\
0\end{array}\right.$} & $\begin{array}{c}1.80 \\
1.87 \\
0\end{array}$ & $\left.\begin{array}{c}0 \\
0 \\
1.57\end{array}\right]$ & $e^{10}$ \\
\hline 3 & 0.5 & & & {$\left[\begin{array}{c}6.97 \\
2.38 \\
0\end{array}\right.$} & $\begin{array}{c}2.08 \\
3.22 \\
0\end{array}$ & $\left.\begin{array}{c}0 \\
0 \\
2.36\end{array}\right]$ & $e^{10}$ \\
\hline
\end{tabular}


4

0.7

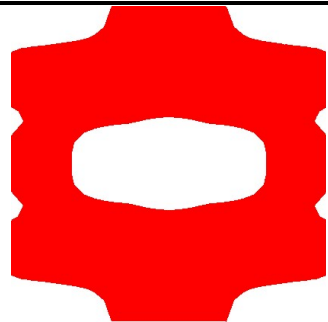

5

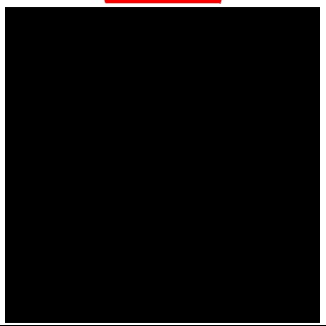

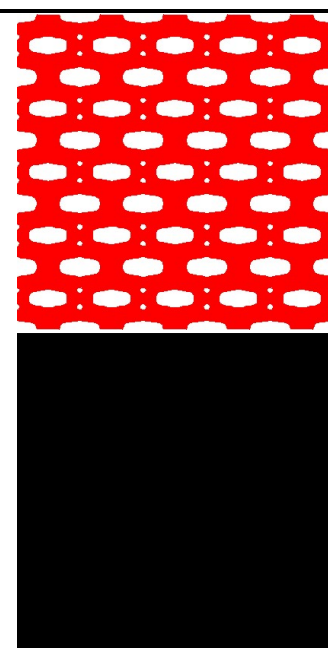

$\left[\begin{array}{ccc}23.07 & 6.92 & 0 \\ 6.92 & 23.07 & 0 \\ 0 & 0 & 8.08\end{array}\right] e^{10}$

Finally, the dynamic multiscale topology optimization design of the clamped beam is displayed in Fig. 9. It can be seen that the optimized topology of the macrostructure comprises five sub different regions plotted with different colors, and each sub region is homogenously configured by the corresponding representative microstructure plotted with the same color. Five distinct material microstructures are listed below the macro topology. Hence, the effectiveness of the dynamic multiscale topology optimization for cellular composites with the multi-regional distributed material microstructures is demonstrated. Moreover, the upper and lower solid face-sheet of the optimized macro topology are featured with solid material microstructures to provide sufficient stiffness and prevent bending deformation. Hence, the FMDO with the generation of the multiregional element densities can provide an appropriate configuration of multiple microstructures within the macrostructure. Additionally, the connectivity between the adjacent material microstructures can be well maintained, owing to the predefined connectors in all material microstructures.

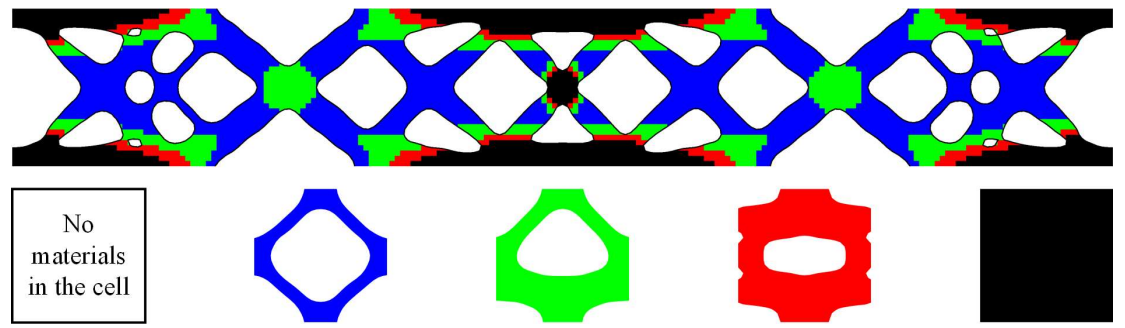

Fig. 9. Multiscale design 1 of the clamped beam

Finally, the convergent histories for the dynamic multiscale topology optimization of the cellular composite are displayed in Fig. 10, in which the objective and the total volume fraction are depicted in Fig. $10(a)$ and the volume fractions of five representative material microstructures are displayed in Fig. $10(b)$. Moreover, the intermediate topologies of both the macrostructure and the material microstructures are displayed in Fig. 10. The iterative curves have a clear, smooth and fast convergence, which displays the high efficiency of the proposed dynamic multiscale design method. 


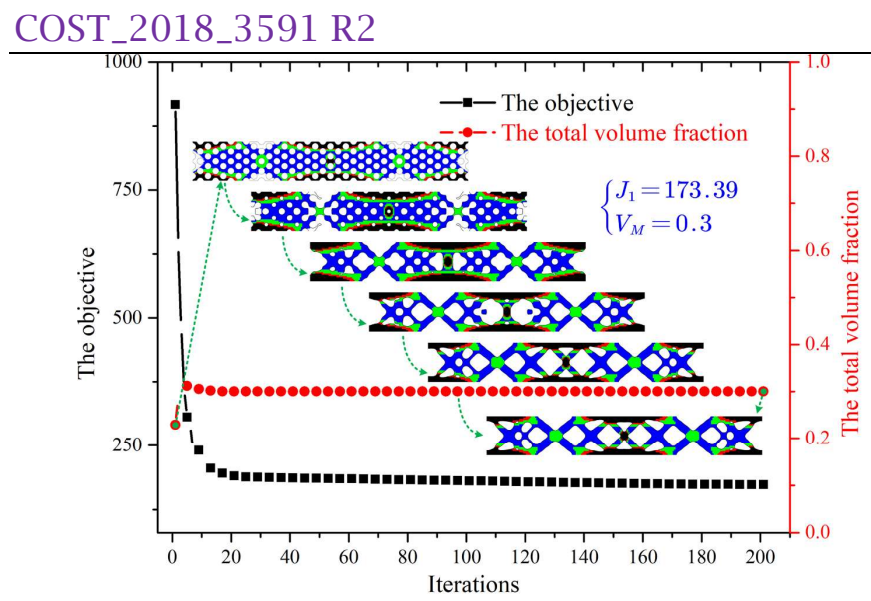

(a) The objective and total volume fraction

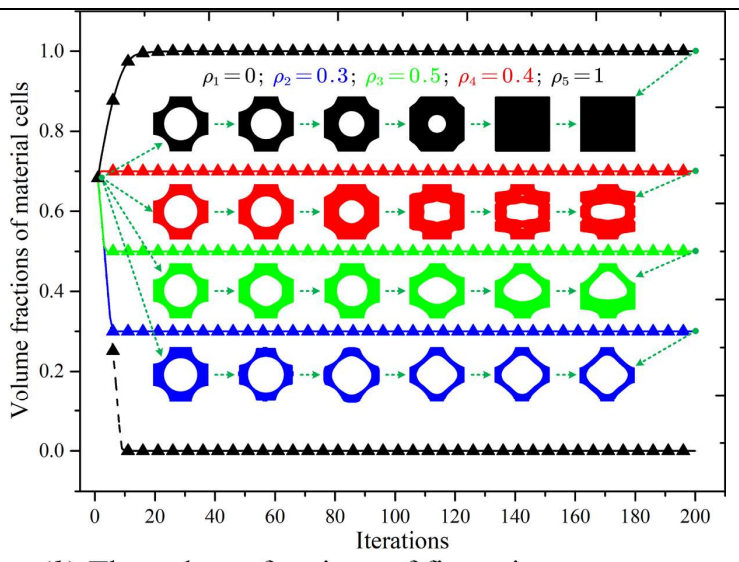

(b) The volume fractions of five microstructures

Fig. 10. Evolution histories.

\subsubsection{Discussions of the Influence of the regularization mechanism}

In this sub section, we discuss the influence of the FMDO on the optimized structural dynamic performance. four schemes (S2-S5) are defined in Table 3 to obtain different regularized distributions. The parameters are consistent with Section 6.1.1, including the structural sizes, the finite element meshes at two scales and so on. The initial continuous distribution of the material element densities still keeps unchanged, as clearly shown in Fig. $6(a)$. The defined S2-S5 schemes are adopted to regularize the element densities, and four regularized distributions are achieved in Fig. 11. It can be easily found that the regularized element densities are also distributed in a multi-regional way, analogously to Fig. 6 (b). Moreover, the macro design domain will be configured by more discrete element densities with a finer group classification.

Table 3. S2-S5 schemes of the regularization mechanism

\begin{tabular}{|c|c|}
\hline S2 & S3 $\quad[00.2),[0.20 .5),[0.50 .8),[0.81]$ \\
\hline S4 & {$[00.2),[0.20 .3),[0.30 .4),[0.40 .5),[0.50 .6),[0.60 .7),[0.70 .8),[0.81]$} \\
\hline S5 & {$[00.1),[0.10 .2),[0.20 .3),[0.30 .4),[0.40 .5),[0.50 .6),[0.60 .7),[0.70 .8),[0.80 .9),[0.91] ;$} \\
\hline
\end{tabular}

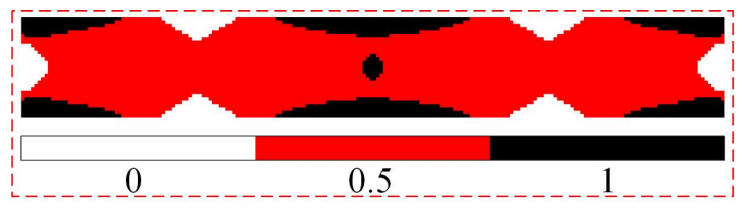

(a) Case 2

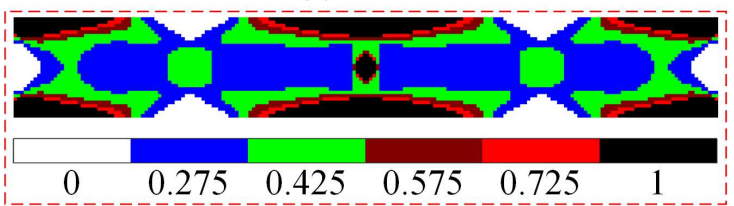

(c) Case 4

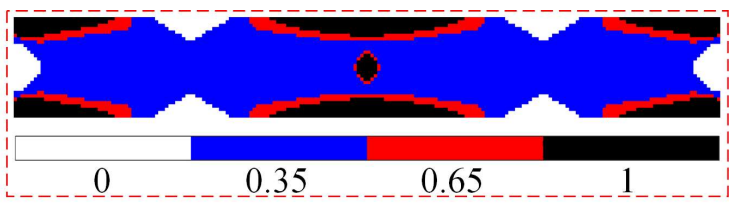

(b) Case 3

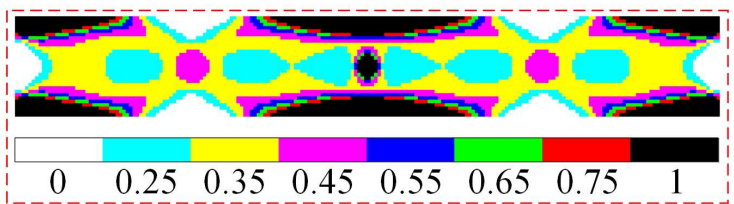

(d) Case 5

Fig. 11 . four regularized distributions

Based on the overall distributions of the regularized element densities in four cases provided in Fig. 11, the concurrent topology optimizations for the macrostructure and multiple representative microstructures are 
performed in four cases. Four dynamic multiscale topology optimization designs of the clamped beam are displayed in Fig. $12(a-d)$. It can be easily seen that the optimized dynamic multiscale designs in four cases are analogous to the result displayed in Fig. 9. That is, the proposed method can realize the optimization of the macro topology, micro topologies and the overall distribution of material microstructures in the macro topology in a unified framework, so that the structural dynamic performance can be improved from these design pillars. Additionally, as we can see, the optimized structural dynamic performance of the multiscale designs in four cases becomes much better with the increasing of the number of the representative material microstructures, namely $J_{2}>J_{3}>J_{4}>J_{5}$. The phenomenon related to the influence of the regularization mechanism is reasonable, owing to the fact that the finer classification of the element densities can generate a denser distribution for multiple microstructures. The design freedom and flexibility of the improvement of the structural dynamic performance are expanded.

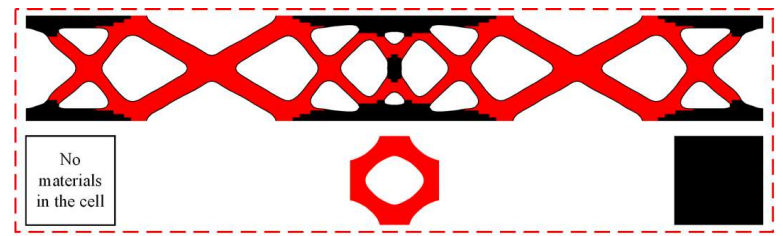

(a) Multiscale design 2: $J_{2}=180.71$

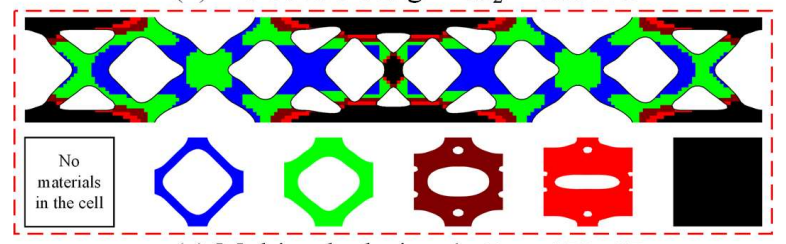

(c) Multiscale design $4: J_{4}=170.67$

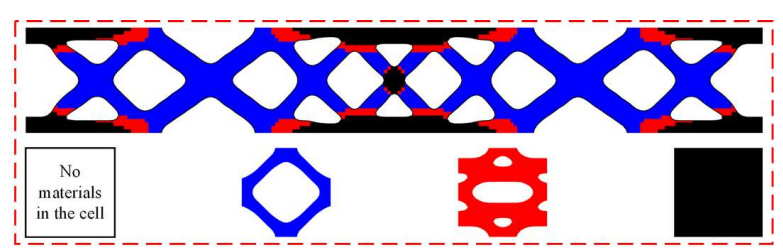

(b) Multiscale design 3: $J_{3}=176.27$

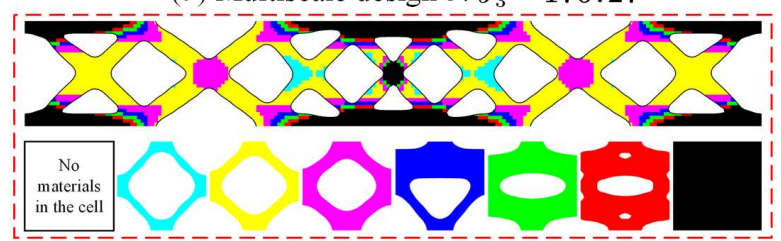

(d) Multiscale design $5: J_{5}=166.35$

Fig. 12. Multiscale designs of the clamped beam

\subsubsection{Comparison with conventional multiscale design}

In order to further show the advantages of the proposed design method, the clamped beam is also optimized by the conventional multiscale topology optimization method, where the macrostructure is configured by only one identical microstructure. The dynamic multiscale topology optimization formulation in Eqs. (13) and (16) can naturally degrade to the conventional multiscale design when the regularized distribution only comprises a unique element density. The design parameters at two scales are consistent with Section 6.1.1. The final multiscale design of the clamped beam is shown in Fig. 13.

It can be seen that only one distinct microstructure and the macrostructure are concurrently optimized in the final design, and the corresponding objective function $\left(J_{0}=688.53\right)$ is much higher than the optimized dynamic compliance in above five cases. Hence, the conventional multiscale design of the clamped beam might be featured with the worst structural dynamic performance. We confirm that the proposed dynamic multiscale topology optimization method can further improve the dynamic performance, and the number 
of multiple microstructures and their overall distribution in the macrostructure have a notable effect on the structural dynamic performance.

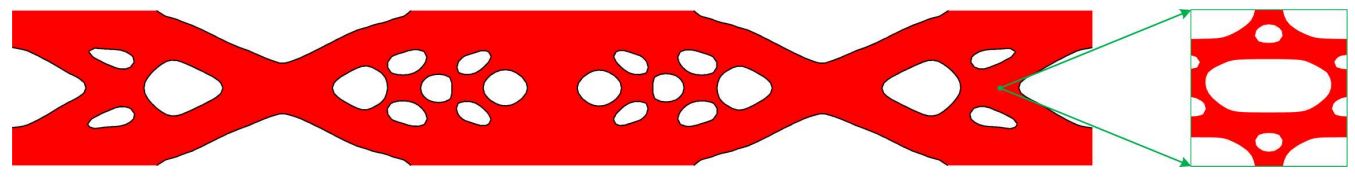

Fig. 13. Conventional multiscale design: $J_{0}=688.53$

\subsection{D supported structure}

In this example, we investigate the effectiveness of the proposed dynamic multiscale topology optimization design method in 3D scenario. In Fig. 14, a 3D supported structure with the dimensions of $L=0.2 \mathrm{~m}, W=0.2 \mathrm{~m}$ and $H=0.15 \mathrm{~m}$ is defined, and four corners located at the bottom surface are fixed. Five downward harmonic dynamic forces $\mathbf{F}=-3 e 5 e^{i 100 t}$ are loaded at the top surface of the $3 \mathrm{D}$ structure. The macrostructure is discretized with $20 \times 20 \times 15$ finite elements, and a mesh of $15 \times 15 \times 15$ finite elements is employed to discretize material microstructures. The maximum volume fractions $V_{M}$ and $V_{d}$ are defined as $25 \%$ and $50 \%$, respectively.

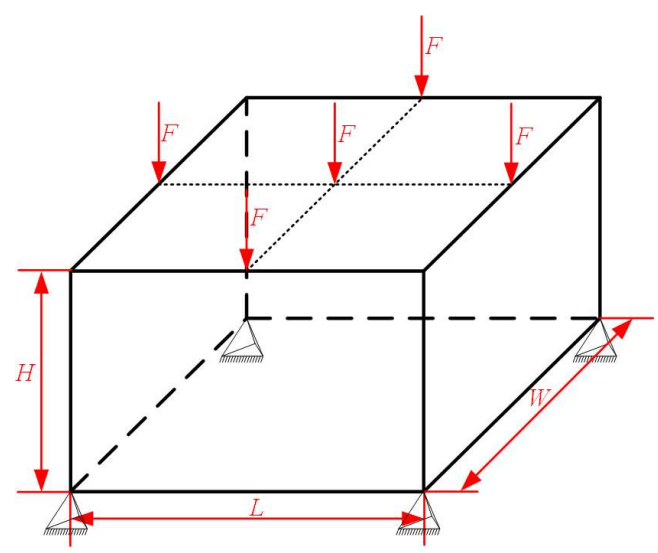

Fig. 14. A 3D supported structure

\subsubsection{Dynamic multiscale topology optimization design}

\section{(a) Stage 1: FMDO}

In Fig. 15, the continuous distribution and the regularized distribution of material densities are respectively displayed. Similar to Fig. 6 (a), the continuous distribution has a large number of the intermediate element densities, which causes the extensive increasing of the microstructures to be devised in the next concurrent topology optimization. It is imperative to introduce the $\mathbf{S 1}$ scheme to regularize material element densities, and we can see that the regularized distribution only has a limited number (5) of discrete element densities, as shown in Fig. $6(b)$. Hence, the macro design domain is divided into five sub regions, and each of which is homogenously distributed by the corresponding discrete element density. 


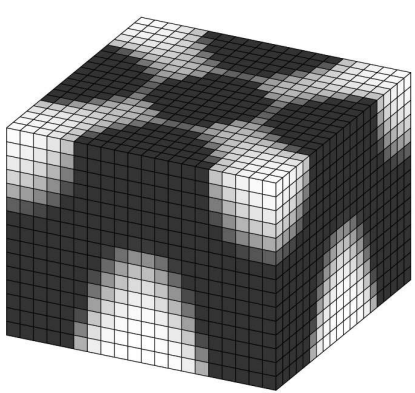

(a) The continuous distribution
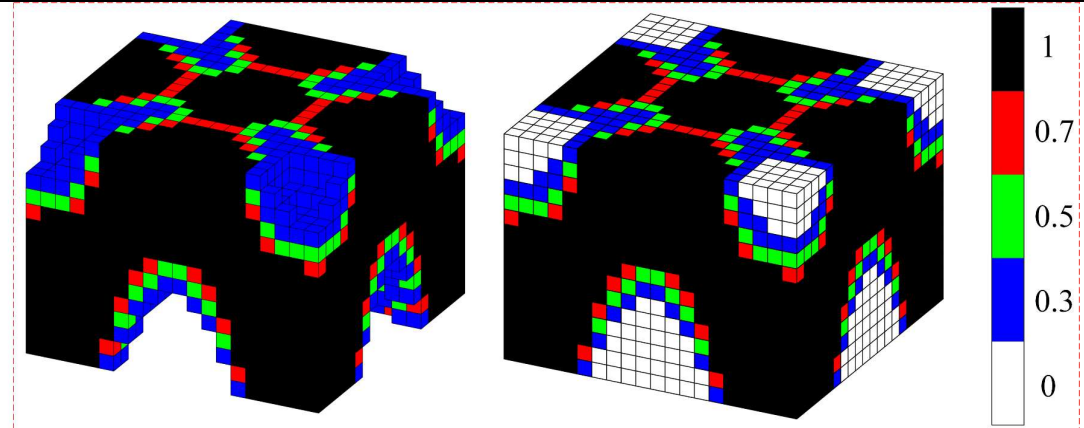

(b) The regularized distribution

Fig. 15. Two distributions in the FMDO

The concurrent topology optimization of the macrostructure and the representative material microstructures is performed, subject to the regularized distribution shown in Fig. $15(b)$. The initial designs at two scales are defined in Fig. 16, where the distinct material microstructures are both evolved starting from the same design in Fig. $16(b)$. As we can see, the two-scale initial designs are both featured with the homogeneously distributed holes, so as to easily to search for the optimal designs. The final topology of the macrostructure is displayed in Fig. 17, which has the smooth boundaries and the clear interface between solids and voids due to the use of the PLSM. Meanwhile, the similar features can also be easily seen in the optimized designs of the representative microstructures, as listed in the third column of Table 4. In order to display the detailed interior information of the micro topologies, the cross-sectional views of four material microstructures are also displayed in Table 4.

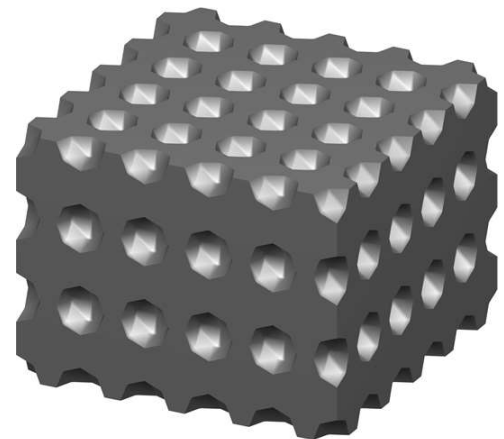

(a) macrostructure

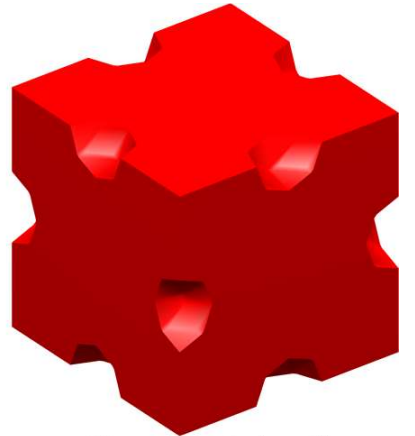

(b) material cell

Fig. 16. Initializations at two scales

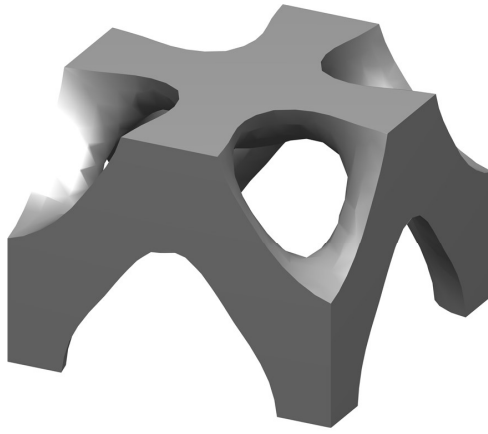

(a) View 1

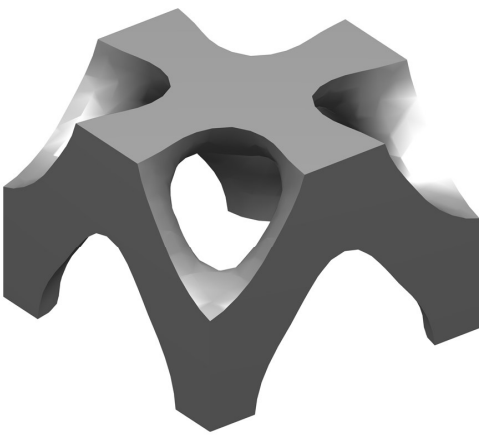

(b) View 2

Fig. 17. The optimized topology of the macrostructure. 
Table 4. The optimized numerical results of material cells

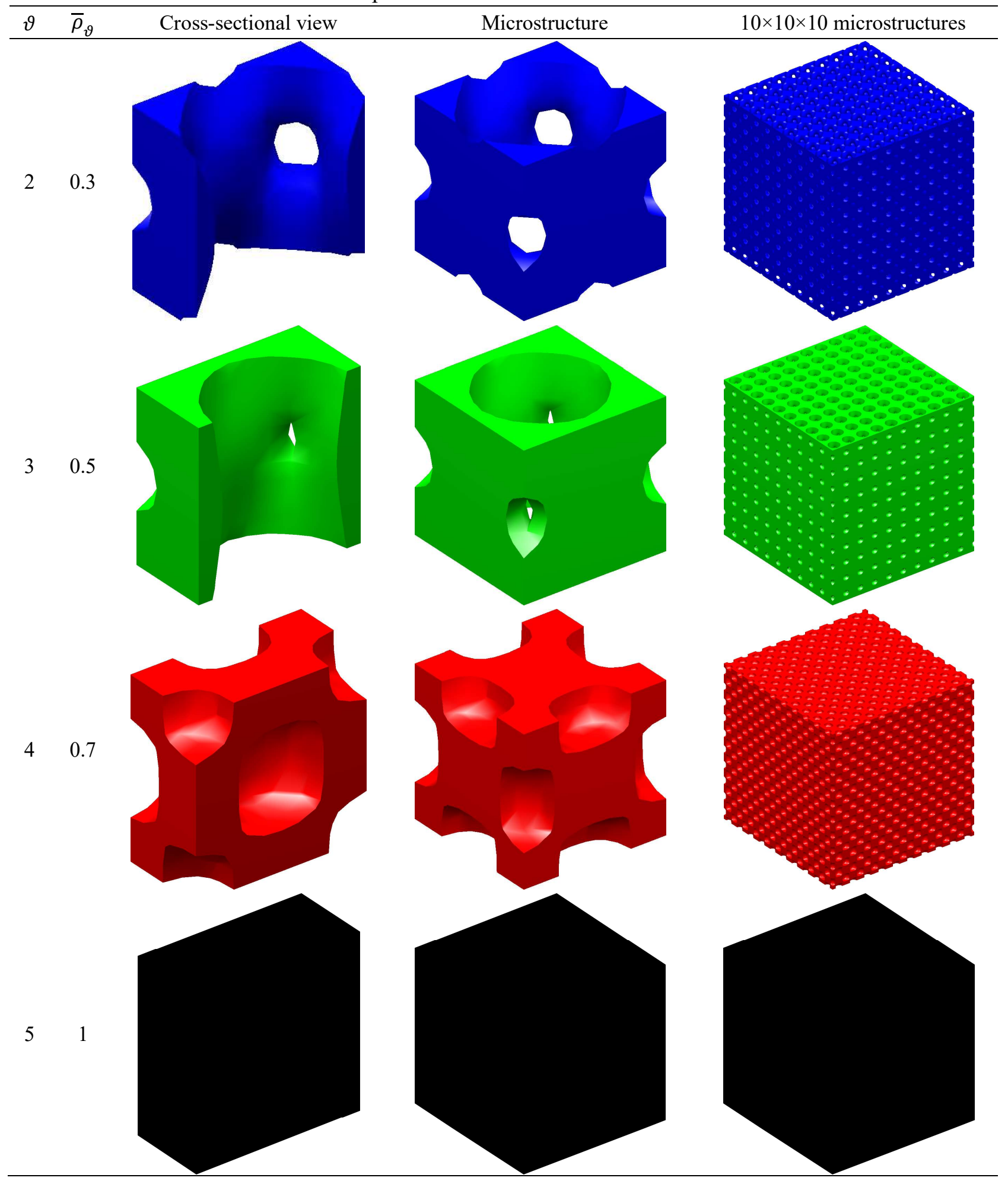

In Fig. 18, the dynamic multiscale topology optimization design of the 3D supported structure is provided, which includes the macro topology, the topologies of five representative microstructures and their overall distribution within the macro topology. Analogously to the 2D design in Section 7.1, the macro topology can be viewed as a combination of all sub macro regions, and each sub region is periodically distributed by a kind of material microstructure with a large number. As defined in the first paragraph of Section 7.2, we 
can easily achieve the normal dimensions of the macro finite element are equal to $1 \mathrm{~cm}$. It is noted that the normal sizes of the material microstructures are $1 \mathrm{~mm}$. Each macro finite element might consist of $10 \times 10 \times$ 10 material microstructures, and the aspect ratio between the macrostructure and the microstructure is much less than 1. The material microstructures with the repetitive pattern are shown in the last column of Table 4. Hence, three pillars in 3D cellular composites, are simultaneously considered in the multiscale topology optimization design to improve the dynamic structural performance.

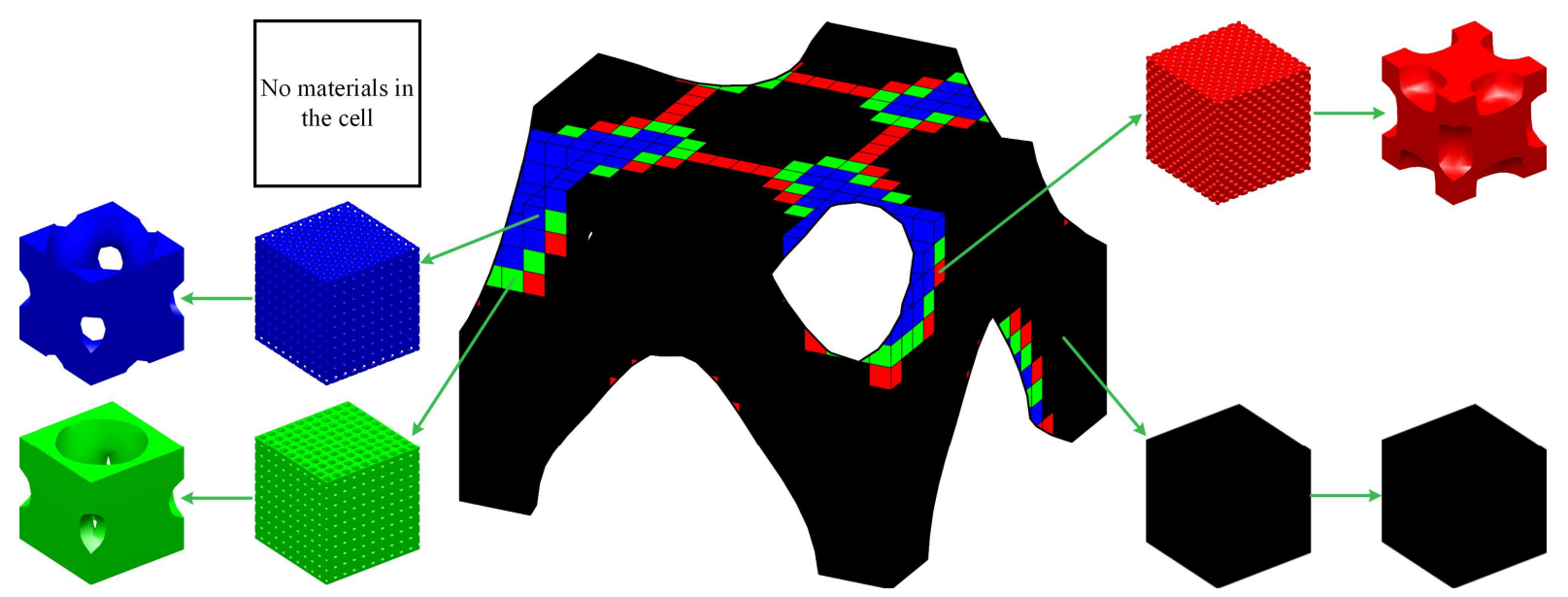

Fig. 18. Multiscale design 1 of the 3D supported structure

Meanwhile, the convergent histories of the objective function and total volume fraction are shown in Fig. $19(a)$, and the volume fractions of five microstructures are depicted in Fig. $19(b)$. The trajectories of the convergent processes illustrate that the objective function and volume fraction can rapidly converge to the optimal solutions within 20 steps and become plateau afterwards, which shows the high efficiency of the proposed dynamic multiscale topology optimization method.

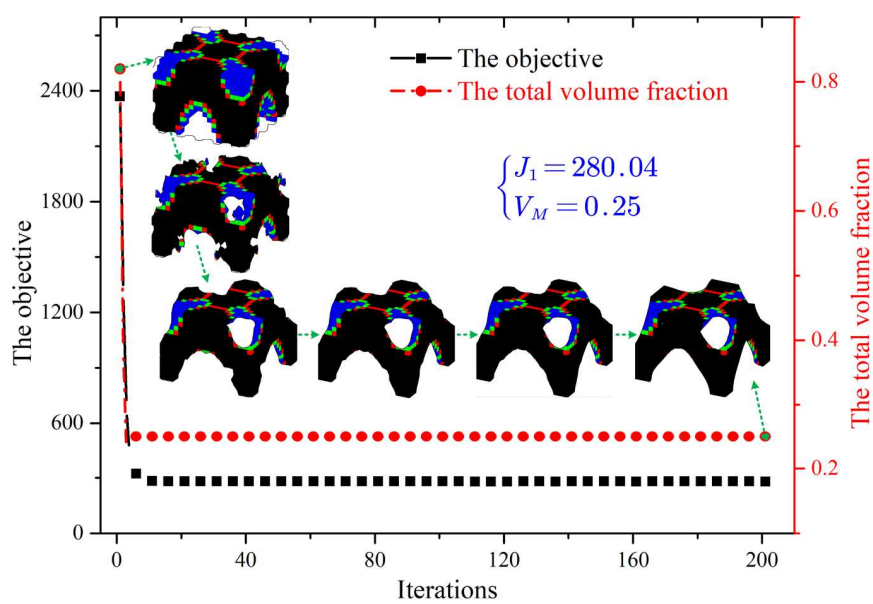

(a) The objective and total volume fraction

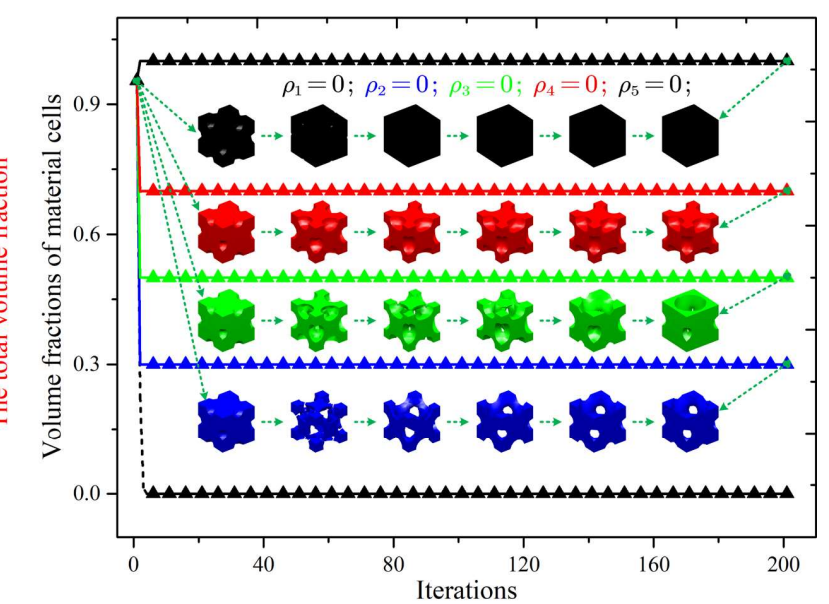

(b) The volume fractions of five microstructures

Fig. 19. Evolution histories 


\subsubsection{Influence of the post-processing mechanism}

In this sub section, the influence of the regularization mechanism in the FMDO on the optimized dynamic performance is also addressed in 3D scenario. The structural design parameters are consistent with Section 7.2.1. S2-S5 schemes defined in Table 3 are respectively applied to regularize the continuously distributed element densities in Fig. 15 (a). Four regularized distributions of the element densities are clearly displayed in Fig. 20. The multi-regional distributed pattern of the element densities is also occurred. That is, the macro design domain is divided into several sub regions and each of them is uniformly configured with the same discrete element densities.

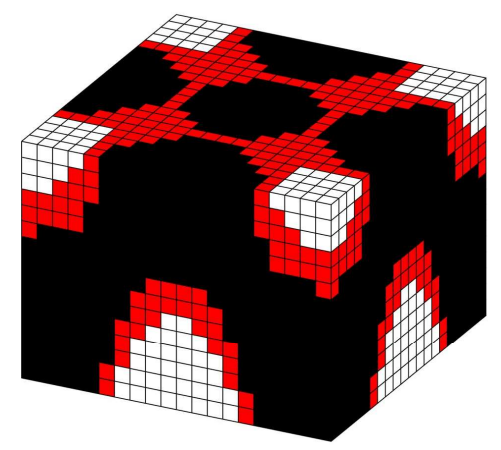

(a) case 2

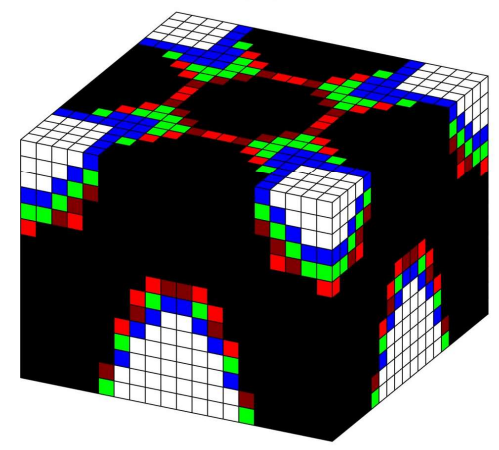

(c) case 4

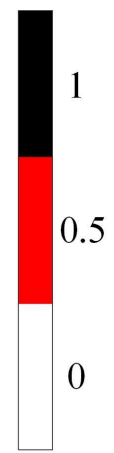

0

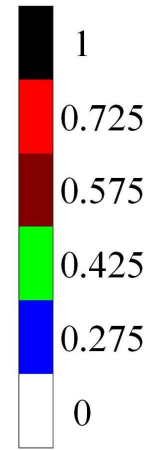

0

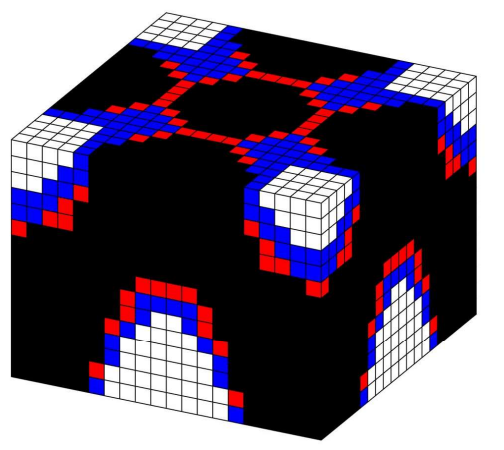

(b) case 3

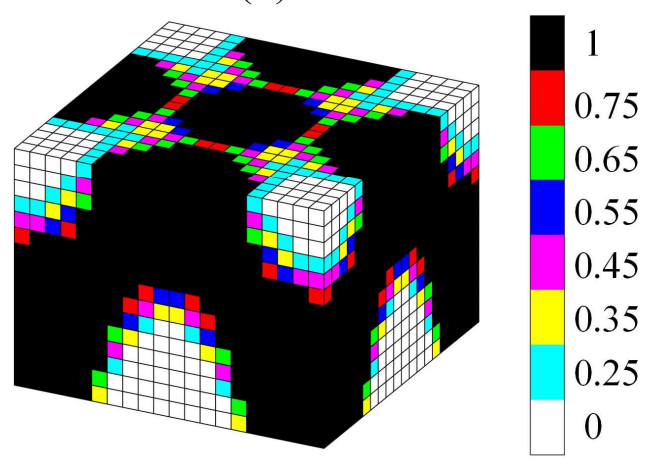

(d) case 5

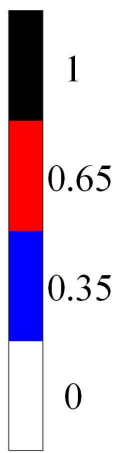

Fig. 20. Four regularized distributions

The concurrent topology optimization for the macrostructure and material microstructures is performed in four cases based on the pre-optimized distributions in the FMDO. Four dynamic multiscale designs of the 3D supported structure are clearly shown in Fig. 21. Meanwhile, the macro topologies in four cases are also listed with the presentation of the multiscale design, as displayed in Fig. 21. It can be easily found that the dynamic performance in four cases is gradually improved with the finer distributions of the microstructures in the design domain, namely $J_{2}>J_{3}>J_{4}>J_{5}$. The main reason originates from that a finer distribution of the representative microstructures can expand the design freedom to enhance the capability to afford the dynamic loads. Hence, the multi-regional distribution of multiple microstructures has a significant impact on the structural dynamic performance. 


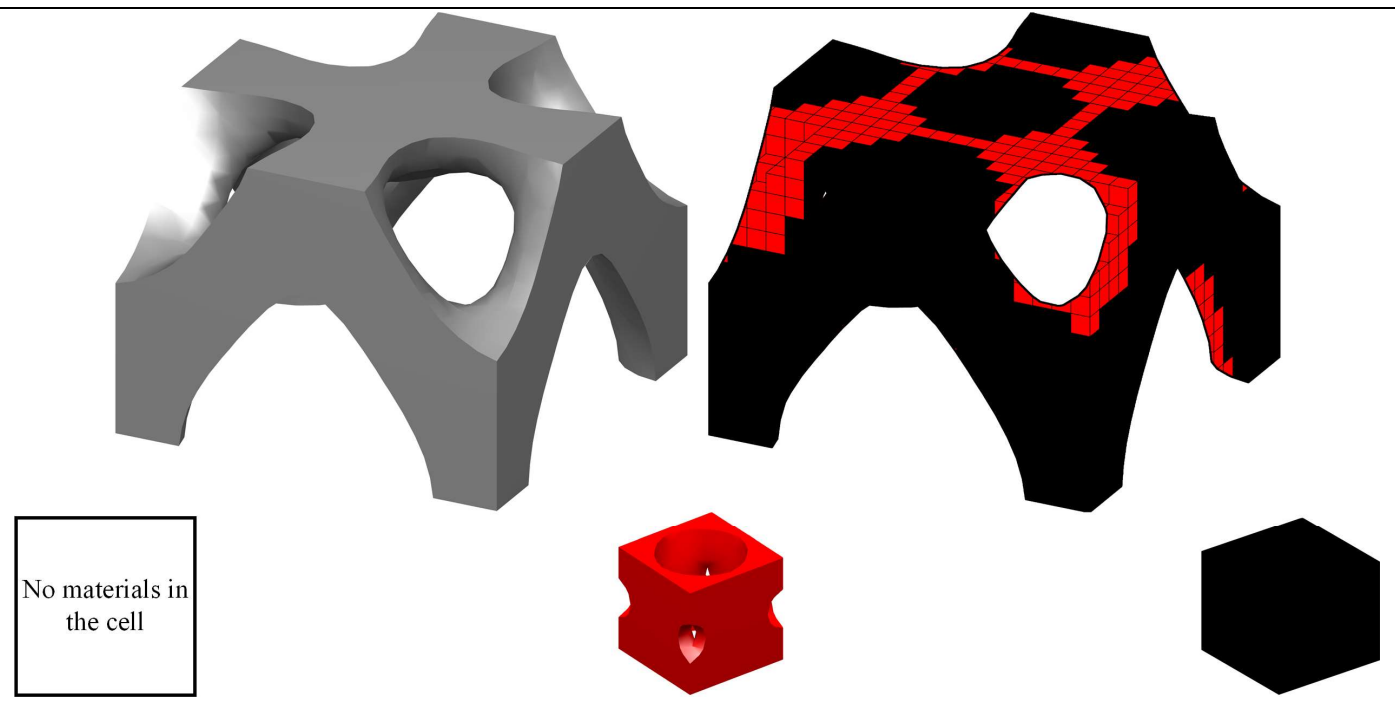

(a) Multiscale design 2: $J_{2}=290.63$

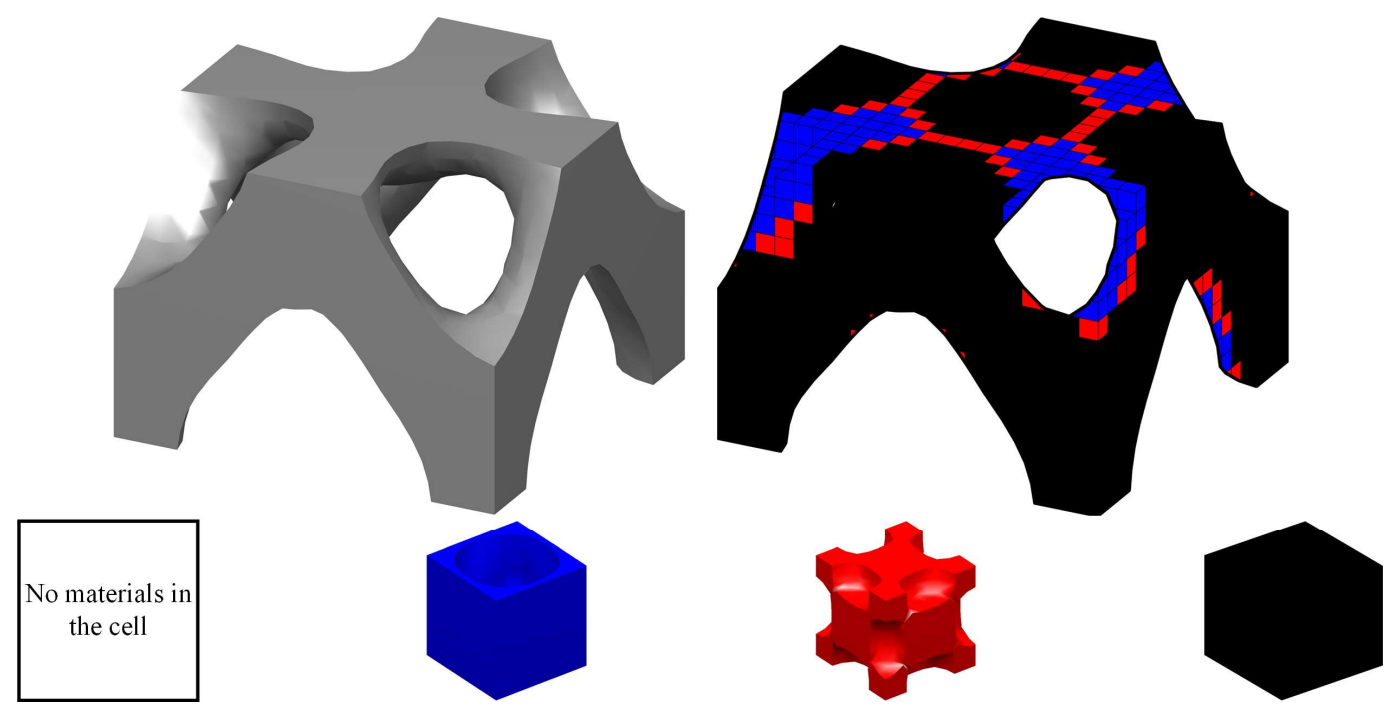

(b) Multiscale design 3: $J_{3}=285.38$

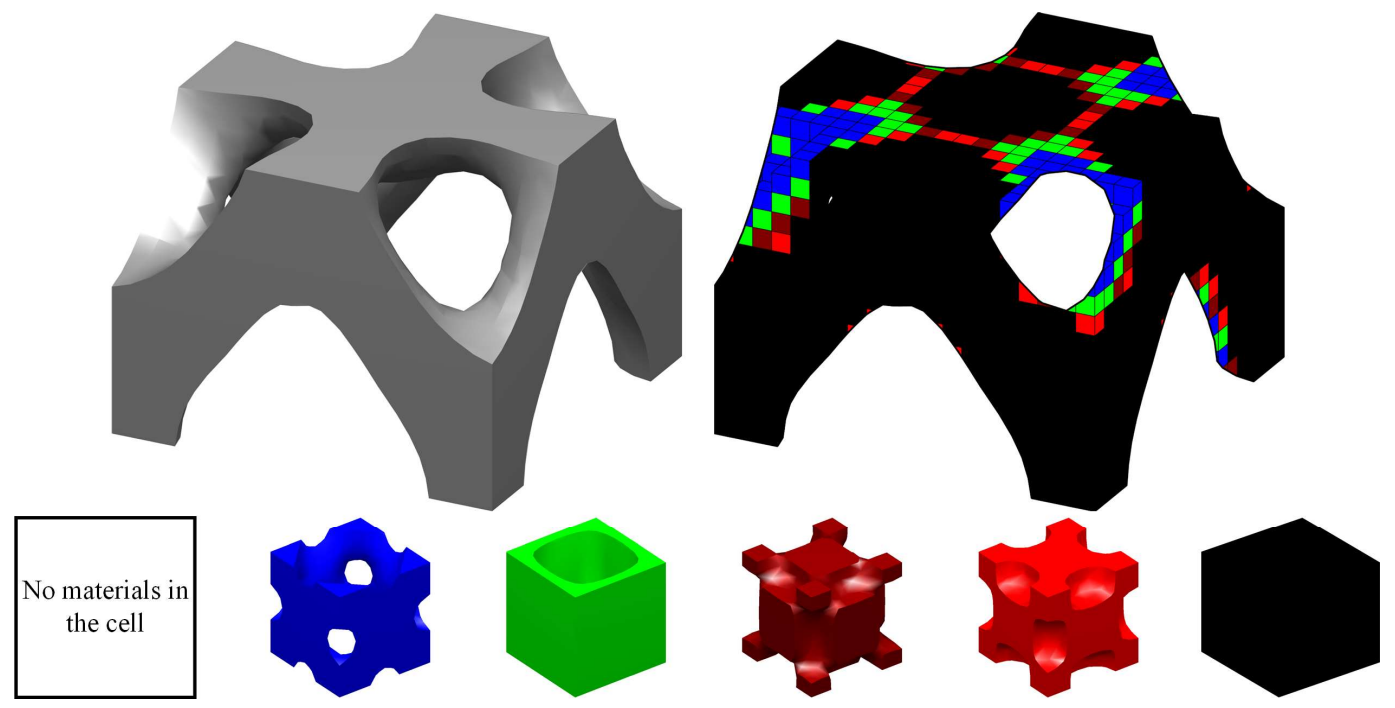

(c) Multiscale design 4: $J_{4}=276.43$ 


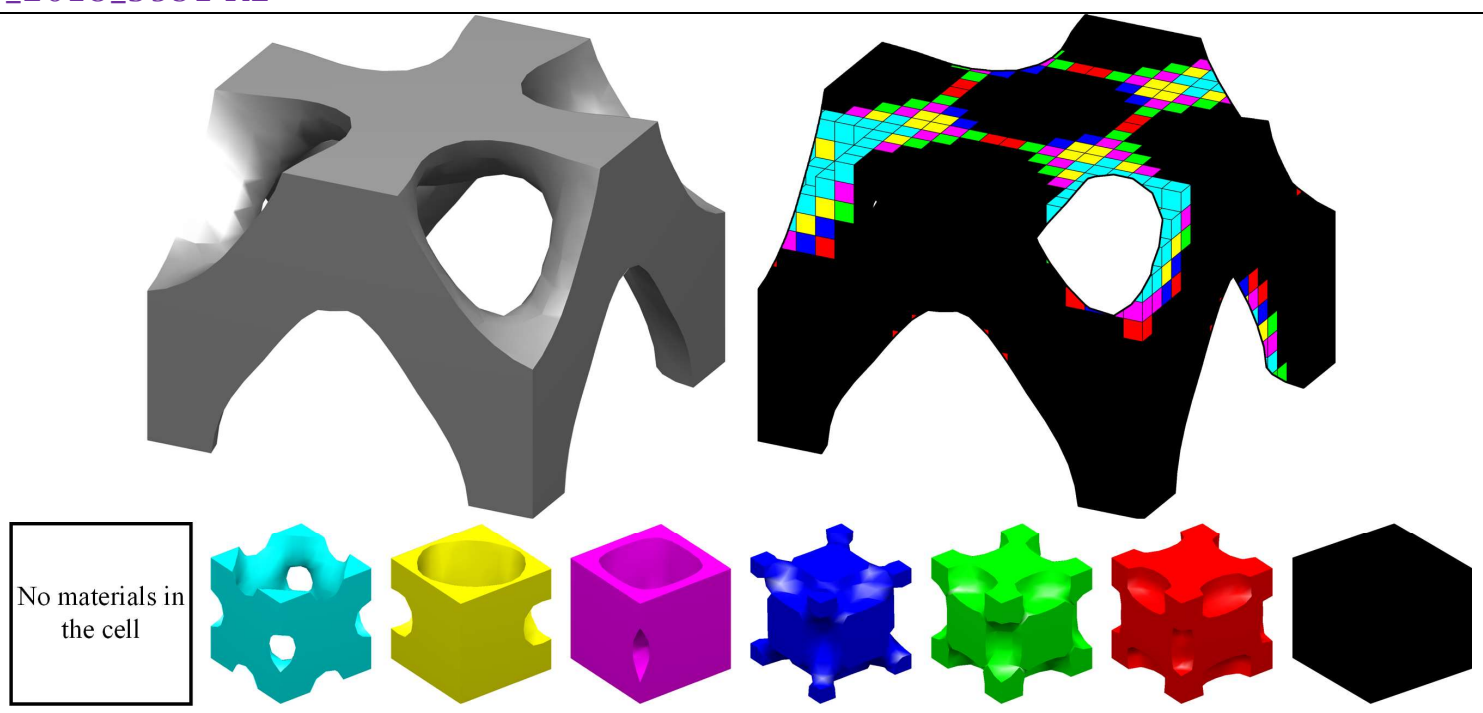

(d) Multiscale design 5: $J_{5}=272.58$

Fig. 21. Multiscale designs of the 3D supported structure in four cases

\subsubsection{Comparison with conventional multiscale design}

Analogously to Section 6.2.3, the conventional multiscale topology optimization design of the 3D supported structure is also addressed to show the superior ability of the current design method. The design parameters keep the same as Section 7.2.1, and the final multiscale design is displayed in Fig. 22. It can be easily found that only a representative microstructure is optimized and periodically distributed in the optimized macro topology. The multiscale design in Fig. 22 only improves the dynamic performance from two design pillars, namely the macro and micro topologies. Hence, the design flexibility to improve the performance might be compromised to a great extent. The phenomenon is also demonstrated by the optimized objective function, that the value is much higher than the above multiscale designs. Hence, the overall distribution for different multiple microstructures is also a key ingredient to improve the structural performance.

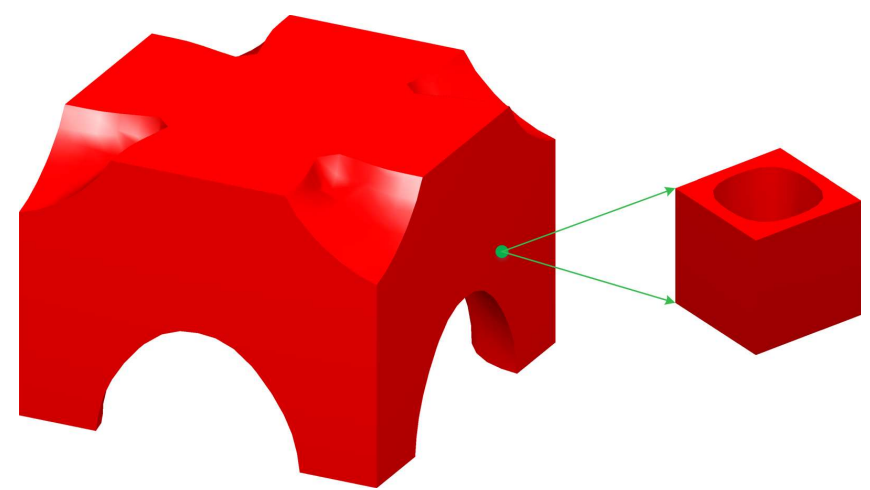

Fig. 22. Conventional multiscale design: $J_{0}=711.34$

\section{Conclusions}

In this study, a new dynamic multiscale topology optimization method that are more effective and efficient is proposed for cellular composites with multiple material microstructures. The numerical implementation 
is mainly involved into two stages. Firstly, the FMDO formulation is developed to generate a distribution of multiple discrete element densities in the macro design domain, which represents the distribution of the distinct microstructures. Secondly, the PLSM combined with the homogenization is applied to develop the concurrent topology optimization formulation.

2D and 3D numerical examples are performed to show the effectiveness of the proposed dynamic topology optimization method. The final multiscale topology optimization designs of cellular composites consist of the topology of the macrostructure, the topologies of the representative microstructures and their overall distribution in the macro topology. Meanwhile, it can be confirmed that the multi-regional distribution of multiple microstructures has a notable effect on the improvement of the structural dynamic performance. The dynamic multiscale topology optimization method can sufficiently make use of the specific functions of the PLSM and FMDO to perfectly serve for cellular composites with multiple microstructures.

Finally, the developed dynamic multiscale topology optimization formulation should be also performed for the meaningful problems in microstructures, like the large deformation and thermal expansion.

\section{Acknowledgments}

This work was supported by the National Basic Scientific Research Program of China (JCKY2016110C012), and the National Natural Science Foundation of China (51705166), and the Australian Research Council (ARC) - Discovery Projects (160102491), and the Financial support from the National Natural Science Foundation of China (51825502).

\section{References}

[1] Gibson LJ, Ashby MF. Cellular solids: structure and properties. Cambridge university press; 1999.

[2] Christensen RM. Mechanics of cellular and other low-density materials. Int J Solids Struct 2000;37:93-104.

[3] Masuda H, Fukuda K. Ordered metal nanohole arrays made by a two-step replication of honeycomb structures of anodic alumina. Science 1995;268:1466-8.

[4] Díaaz AR, Kikuchi N. Solutions to shape and topology eigenvalue optimization problems using a homogenization method. Int J Numer Methods Eng 1992;35:1487-502.

[5] Ma Z-D, Kikuchi N, Hagiwara I. Structural topology and shape optimization for a frequency response problem. Comput Mech 1993;13:157-74.

[6] Bendsøe MP, Kikuchi N. Generating optimal topologies in stuctural design using a homogenization method. Comput Methods Appl Mech Eng 1988;71:197-224.

[7] Zhou M, Rozvany GIN. The COC algorithm, Part II: Topological, geometrical and generalized shape optimization. Comput Methods Appl Mech Eng 1991;89:309-36.

[8] Bendsøe MP, Sigmund O. Material interpolation schemes in topology optimization. Arch Appl Mech 1999;69:635-54.

[9] Xie YM, Steven GP. A simple evolutionary procedure for structural optimization. Comput Struct 1993;49:885-969. 
[10] Sethian JA, Wiegmann A. Structural Boundary Design via Level Set and Immersed Interface Methods. J Comput Phys 2000;163:489-528.

[11] Wang MY, Wang X, Guo D. A level set method for structural topology optimization. Comput Methods Appl Mech Eng 2003;192:227-46.

[12] Allaire G, Jouve F, Toader AM. Structural optimization using sensitivity analysis and a level-set method. J Comput Phys 2004;194:363-93.

[13] Takezawa A, Nishiwaki S, Kitamura M. Shape and topology optimization based on the phase field method and sensitivity analysis. J Comput Phys 2010;229:2697-718.

[14] Gain AL, Paulino GH. Phase-field based topology optimization with polygonal elements: a finite volume approach for the evolution equation. Struct Multidiscip Optim 2012;46:327-42.

[15] Xia Q, Wang MY, Wang S, Chen S. Semi-Lagrange method for level-set-based structural topology and shape optimization. Struct Multidiscip Optim 2006;31:419-29.

[16] Luo Z, Wang M, Wang S, Wei P. A level set based parameterization method for structural shape and topology optimization. Int J Numer Methods Eng 2008;76:1-26.

[17] Luo Z, Tong L. A level set method for shape and topology optimization of large-displacement compliant mechanisms. Int J Numer Methods Eng 2008;76:862-92.

[18] Yamada T, Izui K, Nishiwaki S, Takezawa A. A topology optimization method based on the level set method incorporating a fictitious interface energy. Comput Methods Appl Mech Eng 2010;199:2876-91.

[19] Dunning PD, Alicia Kim H. A new hole insertion method for level set based structural topology optimization. Int J Numer Methods Eng 2013;93:118-34.

[20] Wang Y, Kang Z. A velocity field level set method for shape and topology optimization. Int J Numer Methods Eng 2018;115:1315-36.

[21] Wendland H. Piecewise polynomial, positive definite and compactly supported radial functions of minimal degree. Adv Comput Math 1995;4:389-96.

[22] Rozvany GIN, Bendsøe MP, Kirsch U. Layout optimization of structures. Appl Mech Rev 1995;48:41-119.

[23] Svanberg K. The method of moving asymptotes - a new method for structural optimization. Int J Numer Methods Eng 1987;24:359-73.

[24] Li H, Li P, Gao L, Zhang L, Wu T. A level set method for topological shape optimization of 3D structures with extrusion constraints. Comput Methods Appl Mech Eng 2015;283:615-35.

[25] Wang Y, Luo Z, Zhang N, Kang Z. Topological shape optimization of microstructural metamaterials using a level set method. Comput Mater Sci 2014;87:178-86.

[26] Cadman JE, Zhou S, Chen Y, Li Q. On design of multi-functional microstructural materials. J Mater Sci 2013;48:51-66.

[27] Osanov M, Guest JK. Topology optimization for architected materials design. Annu Rev Mater Res 2016;46:211-33.

[28] Sigmund O. Materials with prescribed constitutive parameters: An inverse homogenization problem. Int J Solids Struct 1994;31:2313-29.

[29] Gao J, Li H, Gao L, Xiao M. Topological shape optimization of 3D micro-structured materials using energy-based homogenization method. Adv Eng Softw 2018;116:89-102.

[30] Gao J, Li H, Luo Z, Gao L, Li P. Topology optimization of micro-structured materials featured with the specific mechanical properties. Int J Comput Methods 2018;15:1850144.

[31] Xia L, Breitkopf P. Design of materials using topology optimization and energy-based homogenization approach in Matlab. Struct Multidiscip Optim 2015;52:1229-41.

[32] Paulino GH, Silva ECN, Le CH. Optimal design of periodic functionally graded composites with prescribed properties. Struct Multidiscip Optim 2009;38:469-89. 
[33] Guest JK, Prévost JH. Optimizing multifunctional materials: design of microstructures for maximized stiffness and fluid permeability. Int J Solids Struct 2006;43:7028-47.

[34] Da D, Yvonnet J, Xia L, Le MV, Li G. Topology optimization of periodic lattice structures taking into account strain gradient. Comput Struct 2018;210:28-40.

[35] Zhou S, Li Q. Design of graded two-phase microstructures for tailored elasticity gradients. J Mater Sci 2008;43:5157.

[36] Rodrigues H, Guedes JM, Bendsoe MP. Hierarchical optimization of material and structure. Struct Multidiscip Optim 2002;24:1-10.

[37] Coelho PG, Fernandes PR, Guedes JM, Rodrigues HC. A hierarchical model for concurrent material and topology optimisation of three-dimensional structures. Struct Multidiscip Optim 2008;35:107-15.

[38] Kato J, Yachi D, Terada K, Kyoya T. Topology optimization of micro-structure for composites applying a decoupling multi-scale analysis. Struct Multidiscip Optim 2014;49:595-608.

[39] Wang Y, Wang MY, Chen F. Structure-material integrated design by level sets. Struct Multidiscip Optim 2016;54:1145-56.

[40] Liu L, Yan J, Cheng G. Optimum structure with homogeneous optimum truss-like material. Comput Struct 2008;86:1417-25.

[41] Zhang WH, Sun SP. Scale-related topology optimization of cellular materials and structures. Int J Numer Methods Eng 2006;68:993-1011.

[42] Belytschko T, Song JH. Coarse-graining of multiscale crack propagation. Int J Numer Methods Eng 2010;81:537-63.

[43] Xia L, Breitkopf P. Concurrent topology optimization design of material and structure within FE 2 nonlinear multiscale analysis framework. Comput Methods Appl Mech Engrg 2014;278:524-42.

[44] Greco F, Leonetti L, Blasi PN. Adaptive multiscale modeling of fiber-reinforced composite materials subjected to transverse microcracking. Compos Struct 2014;113:249-63.

[45] Sivapuram R, Dunning PD, Kim HA. Simultaneous material and structural optimization by multiscale topology optimization. Struct Multidiscip Optim 2016;54:1267-81.

[46] Li H, Luo Z, Zhang N, Gao L, Brown T. Integrated design of cellular composites using a level-set topology optimization method. Comput Methods Appl Mech Eng 2016;309:453-75.

[47] Greco F, Leonetti L, Luciano R, Blasi PN. An adaptive multiscale strategy for the damage analysis of masonry modeled as a composite material. Compos Struct 2016;153:972-88.

[48] Li H, Luo Z, Gao L, Qin Q. Topology optimization for concurrent design of structures with multi-patch microstructures by level sets. Comput Methods Appl Mech Eng 2018;331:536-61.

[49] Gao J, Luo Z, Li H, Gao L. Topology optimization for multiscale design of porous composites with multidomain microstructures. Comput Methods Appl Mech Eng 2019;344:451-76.

[50] Wang Y, Chen F, Wang MY. Concurrent design with connectable graded microstructures. Comput Methods Appl Mech Eng 2017;317:84-101.

[51] Du J, Olhoff N. Topological design of freely vibrating continuum structures for maximum values of simple and multiple eigenfrequencies and frequency gaps. Struct Multidiscip Optim 2007;34:91-110.

[52] Olhoff N, Du J. Generalized incremental frequency method for topological designof continuum structures for minimum dynamic compliance subject to forced vibration at a prescribed low or high value of the excitation frequency. Struct Multidiscip Optim 2016;54:1113-41.

[53] Shu L, Wang MY, Fang Z, Ma Z, Wei P. Level set based structural topology optimization for minimizing frequency response. J Sound Vib 2011;330:5820-34.

[54] Li H, Luo Z, Gao L, Wu J. An improved parametric level set method for structural frequency response optimization problems. Adv Eng Softw 2018;126:75-89. 
[55] Yoon GH. Structural topology optimization for frequency response problem using model reduction schemes. Comput Methods Appl Mech Eng 2010;199:1744-63.

[56] Liu H, Zhang W, Gao T. A comparative study of dynamic analysis methods for structural topology optimization under harmonic force excitations. Struct Multidiscip Optim 2015;51:1321-33.

[57] Niu B, Yan J, Cheng G. Optimum structure with homogeneous optimum cellular material for maximum fundamental frequency. Struct Multidiscip Optim 2009;39:115-32.

[58] Zuo ZH, Huang X, Rong JH, Xie YM. Multi-scale design of composite materials and structures for maximum natural frequencies. Mater Des 2013;51:1023-34.

[59] Xu B, Jiang JS, Xie YM. Concurrent design of composite macrostructure and multi-phase material microstructure for minimum dynamic compliance. Compos Struct 2015;128:221-33.

[60] Kočvara M, Stingl M, Zowe J. Free material optimization: recent progress. Optimization 2008;57:79-100.

[61] Guedes JM, Kikuchi N. Preprocessing and Postprocessing for Materials Based on the Homogenization Method With Adaptive Finite Element Methods. Comput Methods Appl Mech Eng 1990;83:143-98.

[62] Choi KK, Kim N-H. Structural sensitivity analysis and optimization 1: linear systems. Springer Science \& Business Media; 2006.

[63] Osher S, Fedkiw R. Level set methods and dynamic implicit surfaces. vol. 153. Springer Science \& Business Media; 2006.

[64] Gain AL, Paulino GH. A critical comparative assessment of differential equation-driven methods for structural topology optimization. Struct Multidiscip Optim 2013;48:685-710. 